\title{
Highly chemo- and diastereo-selective synthesis of 2,6-diazabicyclo[3.2.0] heptan-7-ones, pyrrolidines and perhydroazirino[2,3-c]pyrroles
}

\author{
Yogesh Kumar, ${ }^{\text {a }}$ Bilash Kulia, ${ }^{\text {a }}$ Prabhpreet Singh, ${ }^{\text {b }}$ and Gaurav Bhargava*a \\ ${ }^{a}$ Department of Chemical Sciences, I. K. Gujral Punjab Technical University, Kapurthala, \\ Punjab 144 603, India \\ ${ }^{b}$ Department of Chemistry, Guru Nanak Dev University, Amritsar, Punjab 143 005, India \\ E-mail:gaurav@ptu.ac.in
}

DOI: https://doi.org/10.24820/ark.5550190.p009.845

\begin{abstract}
The manuscript describes a simple, convenient and metal-free diastereoselective synthesis of 4-halo-3-aryl/alkyl-6-aryl-2,6-diazabicyclo[3.2.0]heptan-7-ones via intramolecular endo-trig haloamination of 3-aminoazetidin-2-ones and its facile transformations to previously unknown methyl 4-halo-3-arylaminopyrrolidine-2-carboxylates and $N$-deprotected diazabicyclo[3.1.0]hexane-2-carboxylic acids in good yields. The synthesis of such heterocyclic system is important in terms of the usefulness as organic synthon as well as their diverse pharmacological profiles.
\end{abstract}

Keywords: Diazabicyclo[3.2.0]heptanones, pyrrolidines, aziridinopyrrolidines, $\beta$-lactams, endotrig haloamination

\section{Introduction}

Over the past decades, $\beta$-lactams have emerged as a useful synthon in organic chemistry. ${ }^{1,2}$ Numerous researchers have explored the synthesis of a variety of novel heterocyclic systems via $\beta$-lactam synthon methodology. ${ }^{3}$ Ojima and his co-workers have described the crucial role of $\beta$-lactam synthon methodology in the synthesis of paclitaxel, docetaxel and new-generation taxoids viz. C-2- and C-3'-modified taxoids, etc. ${ }^{4-6}$ Alcaide et al. have utilized a variety of lactams as organic synthons for the construction of various alkaloid skeletons. ${ }^{7,8}$ Mahajan et al. have explored the $\beta$-lactam synthon approach towards the diastereoselective synthesis of functionalized octahydroisoquinolones, ${ }^{9}$ pyrroloxazine, ${ }^{10}$ tetra/octahydro-isoquinoline ${ }^{11}$ and octahydroindole ${ }^{12}$ ring systems. Literature survey clearly reveals that $\beta$-lactams are important synthons for the synthesis of a variety of useful aza-heterocyclic systems. ${ }^{4-12}$ Functionalized proline esters, the fivemembered azaheterocyclic systems, are important organocatalysts as well as having vital roles in 
biological systems. ${ }^{13-15}$ The perhydroazirino[2,3-c]pyrrole family of natural products has been of interest to the scientific community since their isolation over 50 years ago. ${ }^{16}$ Aziridines are valuable intermediates in natural product synthesis as in the case of the (-)-mesembrine, (-)platynesine, kainoids, sphingosines, epicapreomycidine, actinomycin, $\left( \pm\right.$ )-and feldamycin. ${ }^{17,18}$ Members of this family exhibit potent activity against a variety of cancer cell lines, and were found to be particularly active against solid tumors. ${ }^{19-21}$ In addition, aziridinopyrrolidines have shown interesting biological properties which makes them important synthetic targets. ${ }^{22-25}$ However, the reported methods for preparation of aziridinopyrrolidines are cumbersome and have multistep reaction procedures. ${ }^{17-21}$

Recent publications from our laboratory have reported the synthesis and subsequent transformations of functionalized lactams for the synthesis of (2-oxo-4-styrylazetidin-3yl)pyridine, butadienyl-4-iminomethylazetidin-2-ones, butenylidene-butadienyl-[2,2'-biazetidine]-4,4'-diones, 1,4-benzodiazepin-2-ones and dienyl thiazolidin-4-ones, ${ }^{26-28}$ etc. As a part of our ongoing interest in the synthesis of heterocyclic systems, we have reported earlier the metal free diastereoselective synthesis of diazabicyclo[3.2.0]heptan-7-ones and their transformations to functionalized proline esters. ${ }^{29}$ The reactions were highly diastereo- and chemo-selective and resulted in the formation of diazabicyclo[3.2.0]heptan-7-ones via an endo-trig haloamination reaction. The synthesis of such bicyclic system is important as earlier reports by different workers have revealed their usefulness as type $C \beta$-lactamase inhibitors. ${ }^{30,31}$

The current manuscript summarizes an account of (a) study on halocyclizations of a variety of 3aminoazetidin-2-ones using different haloaminating reagents; (b) a study on mechanistic insight for haloamination reaction using different substituents at nitrogen position; (c) synthetic transformations of diazabicyclo[3.2.0] heptan-7-ones derivatives; (d) lactam mediated synthesis of functionalized proline esters and (e) synthesis of previously unexplored aziridinopyrrolidines. The synthesis of such azaheterocyclic systems especially 4,6-diaryl-3,6-diazabicyclo[3.1.0]hexane-2carboxylic acids are an important in view of their biological properties. Moreover, the earlier effort for the synthesis of $\mathrm{N}$-deprotected 4,6-diaryl-3,6-diazabicyclo[3.1.0]hexane-2-carboxylic acids was unsuccessful. ${ }^{32}$

\section{Results and Discussion}

The starting materials 3-aminoazetidin-2-ones 1, used in halocyclization reactions were prepared by reported methods. ${ }^{33}$ These variably substituted 3-aminoazetidin-2-ones $\mathbf{1}$ were initially investigated for intramolecular ring closure haloamination reactions using different combinations of halogenating reagents and bases in different solvents. The reaction led to the formation of pure 4-halo-3-aryl/alkyl-6-aryl-2,6-diazabicyclo[3.2.0]heptan-7-ones 2a-m (Scheme 1, Table 1). However, the yield of halocyclized products varied with the type of solvent, base and halogen used in the reactions. The reactions were, initially optimized with different halogenation reagents viz. $\mathrm{I}_{2}, \mathrm{Br}_{2}$, NIS, NBS and NCS. The best yield (90\%) was achieved using iodine and potassium 
carbonate as base (Table 1; Entry 2). The halocyclization using NIS and NBS resulted in the formation of 4-halo-3-aryl/alkyl-6-aryl-2,6-diazabicyclo[3.2.0]heptan-7-ones in considerably lower yields. The use of sodium carbonate as base in halocyclization reactions of $\mathbf{1}$ using iodine and bromine resulted in slightly lower yields of the products (Table 1, entries 6-7). When NCS was used as a haloaminating reagent the reactions did not result in the desired product; the starting material remaining intact. The halocyclization was also tested using strong bases i.e. sodium hydride and potassium-t-butoxide. However, this resulted in deterioration of the products.
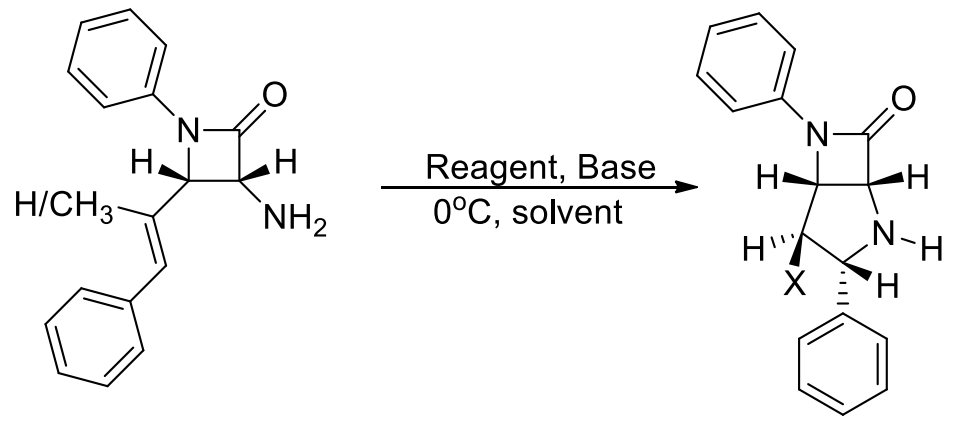

$2 a$

Scheme 1. Halocyclization of 3-aminoazetidin-2-ones $1 \mathbf{a}$.

Table 1. Reaction of $\mathbf{1 a}$ under different reaction conditions

\begin{tabular}{cccccc}
\hline S.No. & Reagent & Base & Solvent & $\begin{array}{c}\text { Reaction } \\
\text { Time }^{\mathrm{a}}\end{array}$ & Yield $(\%)^{\mathrm{b}}$ \\
\hline 1 & $\mathrm{NIS}$ & $\mathrm{K}_{2} \mathrm{CO}_{3}$ & DCM & 30 & 40 \\
2 & $\mathrm{I}_{2}$ & $\mathrm{~K}_{2} \mathrm{CO}_{3}$ & DCM & 90 & 90 \\
3 & $\mathrm{Br}_{2}$ & $\mathrm{~K}_{2} \mathrm{CO}_{3}$ & DCM & 45 & 61 \\
4 & $\mathrm{NBS}$ & $\mathrm{K}_{2} \mathrm{CO}_{3}$ & DCM & 60 & 20 \\
5 & $\mathrm{NCS}$ & $\mathrm{K}_{2} \mathrm{CO}_{3}$ & DCM & 90 & - \\
6 & $\mathrm{I}_{2}$ & $\mathrm{Na}_{2} \mathrm{CO}_{3}$ & DCM & 90 & 80 \\
7 & $\mathrm{Br} 2$ & $\mathrm{Na}_{2} \mathrm{CO}_{3}$ & DCM & 90 & 45 \\
8 & $\mathrm{I}_{2}$ & $t \mathrm{BuOK}_{2}$ & DCM & 90 & - \\
9 & $\mathrm{I}_{2}$ & $\mathrm{NaH}_{2}$ & DCM & 90 & - \\
10 & $\mathrm{I}_{2}$ & $\mathrm{~K}_{2} \mathrm{CO}_{3}$ & DMF & 80 & 55 \\
11 & $\mathrm{I}_{2}$ & $\mathrm{~K}_{2} \mathrm{CO}_{3}$ & THF & 90 & 30 \\
\hline
\end{tabular}

${ }^{a}$ Reaction time in minutes. ${ }^{b}$ Isolated yield after purification. $\mathrm{DCM}=$ dichloromethane

We also studied the effect of a substituent at the alpha position of styryl of 3-aminoazetidin2-ones in these haloamination reactions. The reactions did not give any haloaminations even at high temperature or using harsh reaction conditions, probably due to the steric hindrance at the alpha position of styryl of 3-aminoazetidin-2-ones. 
After optimization of the reaction conditions, diversely substituted 3-aminoazetidin-2-ones 1 were explored in haloaminating reaction with iodine/bromine in the presence of different bases viz. $\mathrm{K}_{2} \mathrm{CO}_{3}$ and $\mathrm{Na}_{2} \mathrm{CO}_{3}$ (Scheme 2). The reactions led to the formation of regio- and diastereoisomerically pure 4-halo-3-aryl/akyl-6-aryl-2,6-diazabicyclo[3.2.0]heptan-7-ones $\mathbf{2}$ in good yields (Table-2; Entries 1-15). There was not much difference in the reactivity as well as yield of the products with changing substituents at the N-1 position of the lactam (Table 2). However the yield of the products in case of bromocyclization is comparatively low (Table 2, Entries 8-13). This is probably due to participation of bromine in side reactions due to its strong acidity.

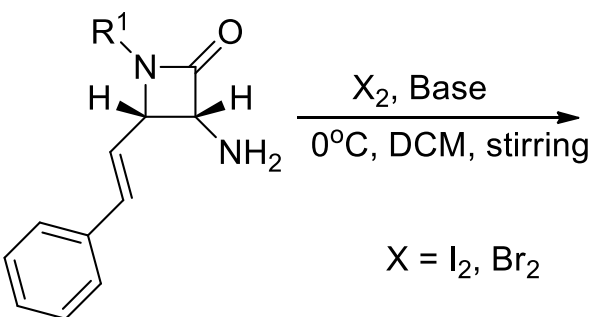
1a, $\mathrm{R}^{1}=-\mathrm{C}_{6} \mathrm{H}_{5}$
1b, $\mathrm{R}^{1}=p-\mathrm{CH}_{3}-\mathrm{C}_{6} \mathrm{H}_{4}$
1c, $\mathrm{R}^{1}=p-\mathrm{Cl}-\mathrm{C}_{6} \mathrm{H}_{4}$
1d, $\mathrm{R}^{1}=p-\mathrm{OMe}-\mathrm{C}_{6} \mathrm{H}_{4}$
1e, $\mathrm{R}^{1}=p-\mathrm{F}-\mathrm{C}_{6} \mathrm{H}_{4}$
1f, $\mathrm{R}^{1}=$ Cyclohexyl
1g, $\mathrm{R}^{1}=$ Benzyl

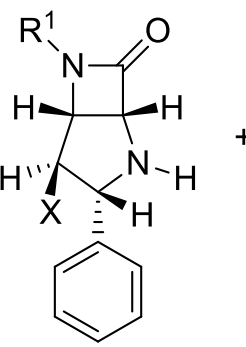

2a, $\mathrm{R}^{1}=-\mathrm{C}_{6} \mathrm{H}_{5} \mathrm{X}=\mathrm{I}$

2b, $\mathrm{R}^{1}=p-\mathrm{CH}_{3}-\mathrm{C}_{6} \mathrm{H}_{4}, \mathrm{X}=\mathrm{I}$

2c, $\mathrm{R}^{1}=p-\mathrm{Cl}-\mathrm{C}_{6} \mathrm{H}_{4}, \mathrm{X}=\mathrm{I}$

2d, $\mathrm{R}^{1}=p-\mathrm{OMe}-\mathrm{C}_{6} \mathrm{H}_{4}, \mathrm{X}=1$

2e, $\mathrm{R}^{1}=p-\mathrm{F}-\mathrm{C}_{6} \mathrm{H}_{4}, \mathrm{X}=$ I

2f, $R^{1}=$ Cyclohexyl, $X=1$

2g, $R^{1}=$ Benzyl, $X=1$

2h, $\mathrm{R}^{1}=-\mathrm{C}_{6} \mathrm{H}_{5}, \mathrm{X}=\mathrm{Br}$

2i, $\mathrm{R}^{1}=p-\mathrm{CH}_{3}-\mathrm{C}_{6} \mathrm{H}_{4}, \mathrm{X}=\mathrm{Br}$

2j, $\mathrm{R}^{1}=p-\mathrm{Cl}-\mathrm{C}_{6} \mathrm{H}_{4}, \mathrm{X}=\mathrm{Br}$

2k, $\mathrm{R}^{1}=p-\mathrm{OMe}-\mathrm{C}_{6} \mathrm{H}_{4}, \mathrm{X}=\mathrm{Br}$

2I, $\mathrm{R}^{1}=p-\mathrm{F}-\mathrm{C}_{6} \mathrm{H}_{4}, \mathrm{X}=\mathrm{Br}$

2m, $R^{1}=$ Benzyl, $X=$ I

Scheme 2. Intramolecular endo-trig-halocyclization of $\mathbf{1}$ for the synthesis of 4-halo-3-aryl/alkyl6-aryl-2,6-diazabicyclo[3.2.0]heptan-7-ones 2.

We next studied the effect of substituents of the participating nitrogen of 3-aminoazetidin-2ones in these haloamination reactions (Scheme 3). Two substituents (i) electron withdrawing (tosyl), (ii) electron donating (methyl) were studied in these haloamination reactions. We have also explored the effect of $N, N$-dimethyl substitution for these haloamination reactions. The reaction of $N$-mono methylated 3-aminoazetidin-2-ones underwent halocyclization in good to fair yield (Table 3; Entries 1-2). However, the reaction of $N$-tosylated 3-aminoazetidin-2-ones did not give any useful product even at high temperature or using harsh reaction conditions. 
Table 2. Synthesis of 4-halo-3-aryl/alkyl-6-aryl-2,6-diazabicyclo[3.2.0]heptan-7-ones 2 by halocyclization reactions

\begin{tabular}{|c|c|c|c|c|c|c|}
\hline S.No. & $\mathrm{R}^{1}$ & $X^{c}$ & Base & Product & $\begin{array}{c}\text { Reaction } \\
\text { Time }^{a}\end{array}$ & $\begin{array}{l}\text { Yield } \\
(\%)^{b}\end{array}$ \\
\hline 1 & $\mathrm{C}_{6} \mathrm{H}_{5}$ & I & $\mathrm{K}_{2} \mathrm{CO}_{3}$ & $\mathbf{2 a}$ & 90 & 90 \\
\hline 2 & $p-\mathrm{CH}_{3}-\mathrm{C}_{6} \mathrm{H}_{4}$ & I & $\mathrm{K}_{2} \mathrm{CO}_{3}$ & $2 b$ & 90 & 82 \\
\hline 3 & $p-\mathrm{Cl}-\mathrm{C}_{6} \mathrm{H}_{4}$ & I & $\mathrm{K}_{2} \mathrm{CO}_{3}$ & $2 c$ & 90 & 65 \\
\hline 4 & $p-\mathrm{CH}_{3} \mathrm{O}-\mathrm{C}_{6} \mathrm{H}_{4}$ & I & $\mathrm{K}_{2} \mathrm{CO}_{3}$ & 2d & 90 & 66 \\
\hline 5 & $p-\mathrm{F}-\mathrm{C}_{6} \mathrm{H}_{4}$ & I & $\mathrm{K}_{2} \mathrm{CO}_{3}$ & $2 e$ & 90 & 62 \\
\hline 6 & cyclohexyl & I & $\mathrm{K}_{2} \mathrm{CO}_{3}$ & $2 f$ & 90 & 75 \\
\hline 7 & Benzyl & I & $\mathrm{K}_{2} \mathrm{CO}_{3}$ & $2 g$ & 90 & 60 \\
\hline 8 & $\mathrm{C}_{6} \mathrm{H}_{5}$ & $\mathrm{Br}$ & $\mathrm{K}_{2} \mathrm{CO}_{3}$ & $2 \mathrm{~h}$ & 45 & 61 \\
\hline 9 & $p-\mathrm{CH}_{3}-\mathrm{C}_{6} \mathrm{H}_{4}$ & $\mathrm{Br}$ & $\mathrm{K}_{2} \mathrm{CO}_{3}$ & $2 \mathbf{i}$ & 45 & 55 \\
\hline 10 & $p-\mathrm{Cl}-\mathrm{C}_{6} \mathrm{H}_{4}$ & $\mathrm{Br}$ & $\mathrm{K}_{2} \mathrm{CO}_{3}$ & $2 \mathbf{j}$ & 45 & 50 \\
\hline 11 & $p-\mathrm{CH}_{3} \mathrm{O}-\mathrm{C}_{6} \mathrm{H}_{4}$ & $\mathrm{Br}$ & $\mathrm{K}_{2} \mathrm{CO}_{3}$ & $2 k$ & 45 & 55 \\
\hline 12 & $p-\mathrm{F}-\mathrm{C}_{6} \mathrm{H}_{4}$ & $\mathrm{Br}$ & $\mathrm{K}_{2} \mathrm{CO}_{3}$ & 21 & 45 & 60 \\
\hline 13 & Benzyl & $\mathrm{Br}$ & $\mathrm{K}_{2} \mathrm{CO}_{3}$ & $2 \mathrm{~m}$ & 45 & 45 \\
\hline 14 & $\mathrm{C}_{6} \mathrm{H}_{5}$ & I & $\mathrm{Na}_{2} \mathrm{CO}_{3}$ & $2 a$ & 90 & 80 \\
\hline 15 & $p-\mathrm{CH}_{3}-\mathrm{C}_{6} \mathrm{H}_{4}$ & I & $\mathrm{Na}_{2} \mathrm{CO}_{3}$ & $2 \mathbf{b}$ & 90 & 75 \\
\hline
\end{tabular}

${ }^{\mathrm{a}}$ Reaction time in minutes. $\quad{ }^{\mathrm{b}}$ Isolated yield after purification. $\quad{ }^{\mathrm{c}} 1.2$ equivalent.

The reaction of $N, N$-disubstituted as well as mono $N^{5}$-tosylatedazetidin-2-one did not provide any desired product and only led to the recovery of the starting material, even after several hours of stirring at different temperatures using even higher amounts of iodine/bromine or using different bases, such as potassium carbonate, sodium carbonate, sodium hydride and potassium-t-butoxide. From these experimental observations, it may be concluded that the endo-trig haloamination reaction was not observed in the presence of an electron withdrawing group at the $\mathrm{N}$-position and that the reaction is facilitated by the presence of an electron donating group. However, the reaction of $\mathbf{5 b}$ and $\mathbf{5 c}$ was not observed due to the more steric crowding for endo-trig haloamination reaction. 

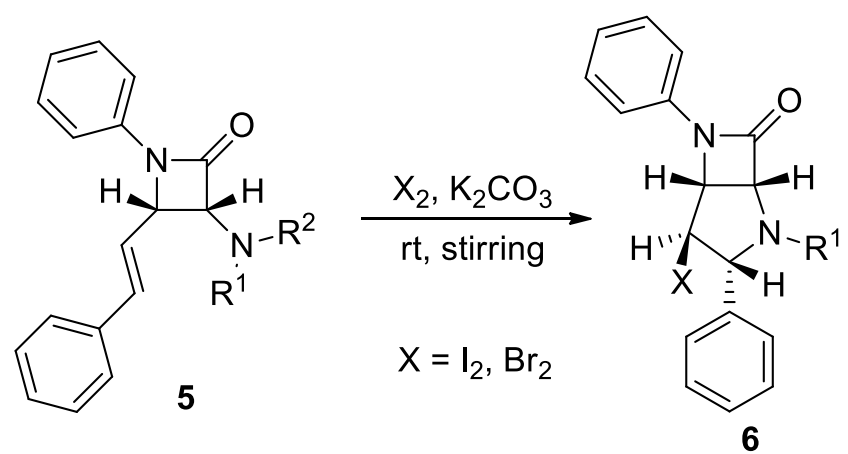

$$
\begin{aligned}
& \text { 5a, } R^{1}=\mathrm{CH}_{3}, \mathrm{R}^{2}=\mathrm{H} \\
& \text { 5b, } \mathrm{R}^{1}=\mathrm{CH}_{3}, \mathrm{R}^{2}=\mathrm{CH}_{3} \\
& \text { 5c, } \mathrm{R}^{1}=p \text {-tosyl, } \mathrm{R}^{2}=\mathrm{H}
\end{aligned}
$$

6a, $\mathrm{R}^{1}=\mathrm{CH}_{3}, \mathrm{R}^{2}=\mathrm{H} ; \mathrm{X}=\mathrm{I}$

6b, $\mathrm{R}^{1}=\mathrm{CH}_{3}, \mathrm{R}^{2}=\mathrm{H} ; \mathrm{X}=\mathrm{Br}$

Scheme 3. 4-Halo-2-methyl-3,6-diphenyl-2,6-diazabicyclo[3.2.0] heptan-7-ones 6.

Table 3. 4-Halo-2-methyl-3,6-diphenyl-2,6-diazabicyclo[3.2.0]heptan-7-ones 6

\begin{tabular}{ccccccc}
\hline S. No. & $\mathrm{R}^{1}$ & $\mathrm{R}^{2}$ & $\mathrm{X}$ & Product & $\begin{array}{c}\text { Reaction } \\
\text { Time }^{\mathrm{a}}\end{array}$ & \begin{tabular}{c} 
Yield $(\%)^{\mathrm{b}}$ \\
\hline 1
\end{tabular} \\
$\mathrm{CH}_{3}$ & $\mathrm{H}$ & $\mathrm{I}$ & $\mathbf{6 a}$ & 90 & 75 \\
2 & $\mathrm{CH}_{3}$ & $\mathrm{H}$ & $\mathrm{Br}$ & $\mathbf{6 b}$ & 50 & 40 \\
3 & $\mathrm{CH}_{3}$ & $\mathrm{CH}_{3}$ & $\mathrm{I}$ & $\mathbf{6 c}$ & 90 & 0 \\
4 & $p$-tosyl & $\mathrm{H}$ & $\mathrm{I}$ & $\mathbf{6 d}$ & 90 & 0 \\
\hline
\end{tabular}

${ }^{\mathrm{a}}$ Reaction time in minutes. $\quad{ }^{\mathrm{b}}$ Isolated yield after purification.

The diastereomerically pure, functionalized novel 4-halo-3-aryl/alkyl-6-aryl-2,6-diazabicyclo[3.2.0]heptan-7-ones 2, thus obtained were characterized on the basis of analytical and spectral evidence. The compound, 4-iodo-3,6-diphenyl-2,6-diazabicyclo[3.2.0]heptan-7-one 2a for example, analyzed for $\mathrm{C}_{18} \mathrm{H}_{17} \mathrm{IN}_{2} \mathrm{O}$ showed a molecular ion peak at $m / z, 391(\mathrm{M}+1)$ in its mass spectrum. Its IR spectrum showed strong absorption peaks at $1755 \mathrm{~cm}^{-1}$ corresponding to the carbonyl group of a azetidin-2-one. The ${ }^{1} \mathrm{H}$ NMR $(300 \mathrm{MHz})$ spectrum showed a characteristic doublet at $\delta 4.91$ having $J 3.6 \mathrm{~Hz}$ corresponding to $\mathrm{H}_{1}$ proton of the ring, an unresolved doublet of doublet at $\delta 4.94$ having $J 3.6 \mathrm{~Hz}$ corresponding to $\mathrm{H}_{5}$ of the lactam ring, a multiplet at $\delta 5.02$ corresponding to $\mathrm{H}_{3} \& \mathrm{H}_{4}$ protons. The ${ }^{13} \mathrm{C}$ NMR have shown the presence of one carbonyl carbon at $\delta 164.2$ and four aliphatic carbons at $\delta 30.7,67.2,71.80$, and $\delta 74.7$ corresponding to C-4, C-5, C-1 and C-3 respectively. The relative stereochemistry of the different ring protons has been established with the help of earlier report. ${ }^{29}$ The 4-iodo-3,6-diphenyl-2,6diazabicyclo[3.2.0] heptan-7-one (2a) has shown the anti stereochemistry between $\mathrm{H}^{5}$ of azetidin2-one and $\mathrm{H}^{4}$ of the pyrrole ring (Figure 1). 


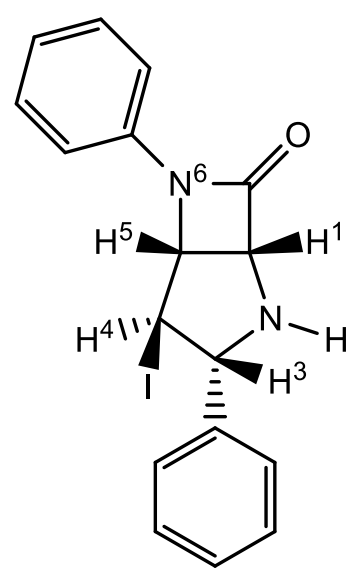

Figure 1. 4-Iodo-3,6-diphenyl-2,6-diazabicyclo[3.2.0]heptan-7-one 2a.

A proposed mechanism for the formation of azabicyclo[3.2.0]heptanes involves the initial coordination of halogen to the double bond at C-4 position of $\beta$-lactam leading to formation of a halonium ion. This is followed by a nucleophilic attack of nitrogen attached to $\mathrm{C}-3$ position of lactam ring to the C-6 position of halonium ion (Scheme-4) thereby yielding corresponding diazabicyclo[3.2.0]heptanes $\mathbf{2}$ in good yields.

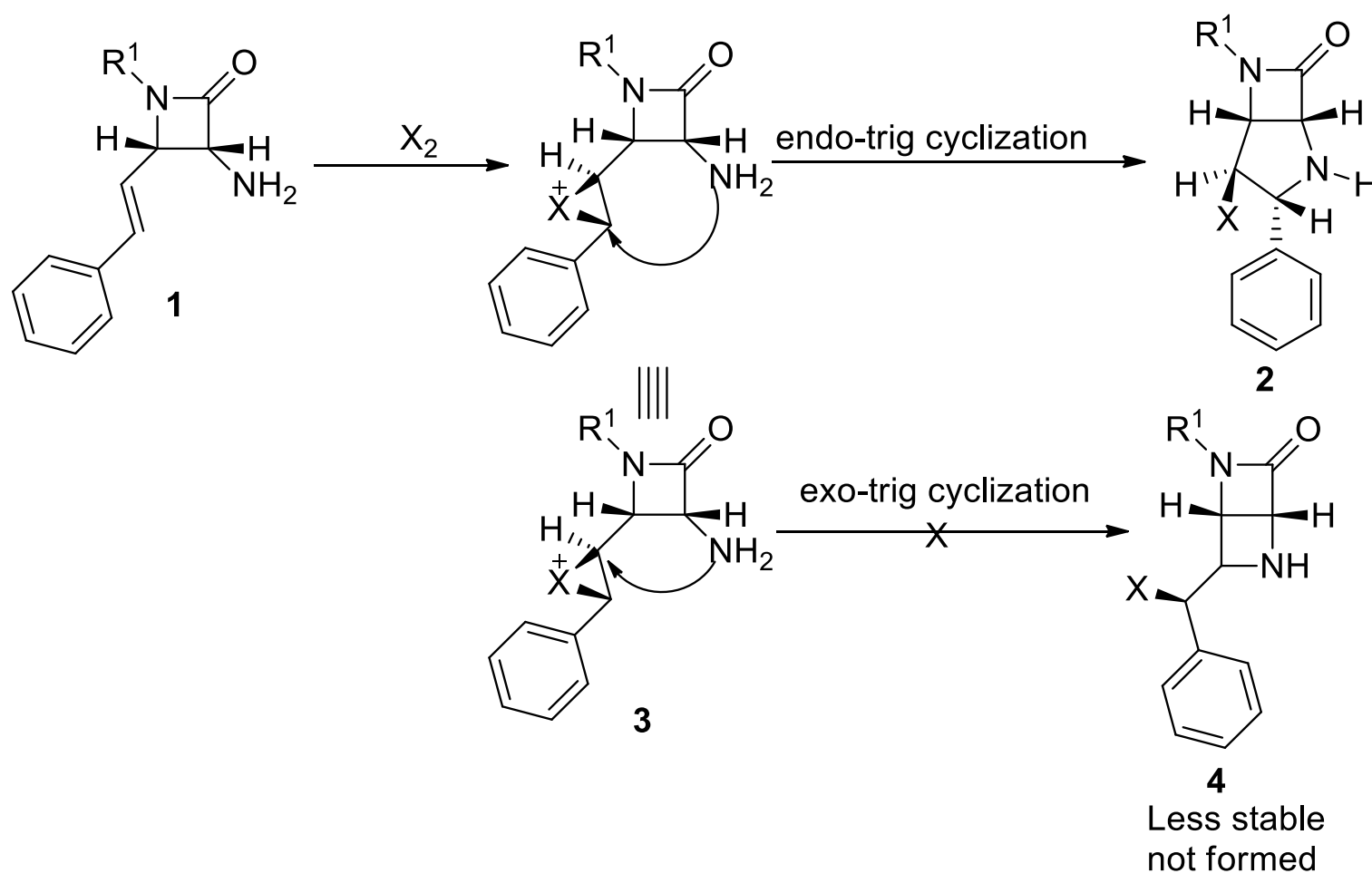

Scheme 4. A plausible mechanism depicting the formation of 4- halo-3-phenyl-6-aryl-2,6-diazabicyclo[3.2.0]heptan-7-ones 
The 4-halo-3-aryl/alkyl-6-aryl-2,6-diazabicyclo[3.2.0]heptan-7-ones 2 were explored for the synthesis of pyrrolidine esters by amidolytic ring hydrolysis of $\mathrm{N}^{6}-\mathrm{C}^{7}$ bond using different bases viz. sodium alkoxide. The reaction resulted in the formation of 4-halo-5-phenyl-3-arylaminopyrrolidine-2-carboxylic acid methyl esters 7 in excellent yields (90\%; Scheme-5, Table 4).

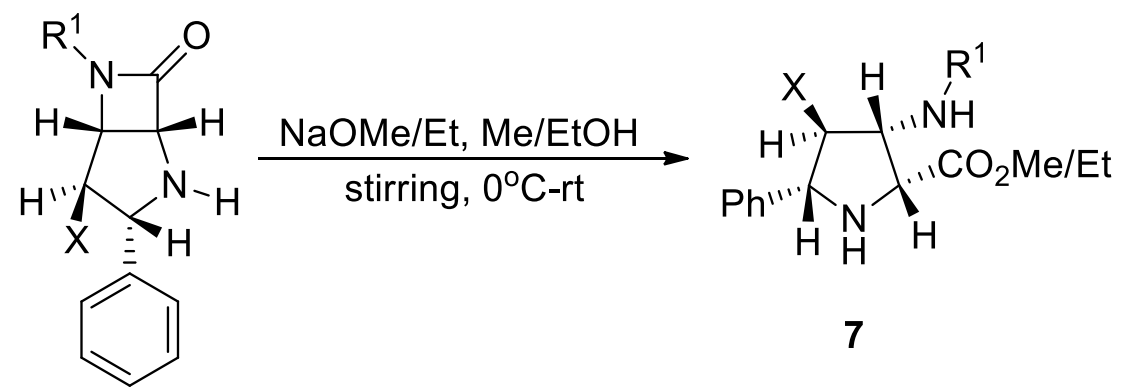

2

Scheme 5. Synthesis of alkyl 4-iodo-5-aryl-3-(arylamino)pyrrolidine-2-carboxylates 7.

Table 4. Alkyl 4-iodo-5-aryl-3-(arylamino)pyrrolidine-2-carboxylates 7

\begin{tabular}{ccccccc}
\hline S.No. & $\mathrm{R}^{1}$ & $\mathrm{X}$ & Base & Solvent & Product $^{\mathrm{a}}$ & $\begin{array}{c}\text { Yield } \\
(\%)^{\mathrm{b}}\end{array}$ \\
\hline 1 & $\mathrm{C}_{6} \mathrm{H}_{5}$ & $\mathrm{I}$ & $\mathrm{NaOCH}_{3}$ & $\mathrm{CH}_{3} \mathrm{OH}$ & $\mathbf{7 a}$ & 85 \\
2 & $p-\mathrm{CH}_{3} \mathrm{C}_{6} \mathrm{H}_{4}$ & $\mathrm{I}$ & $\mathrm{NaOCH}_{3}$ & $\mathrm{CH}_{3} \mathrm{OH}$ & $\mathbf{7 b}$ & 88 \\
3 & $p-\mathrm{ClC}_{6} \mathrm{H}_{4}$ & $\mathrm{I}$ & $\mathrm{NaOCH}_{3}$ & $\mathrm{CH}_{3} \mathrm{OH}$ & $\mathbf{7 c}$ & 75 \\
4 & $p-\mathrm{CH}_{3} \mathrm{OC}_{6} \mathrm{H}_{4}$ & $\mathrm{I}$ & $\mathrm{NaOCH}_{3}$ & $\mathrm{CH}_{3} \mathrm{OH}$ & $\mathbf{7 d}$ & 79 \\
5 & $\mathrm{C}_{6} \mathrm{H}_{5}$ & $\mathrm{Br}$ & $\mathrm{NaOCH}_{3}$ & $\mathrm{CH}_{3} \mathrm{OH}$ & $\mathbf{7 e}$ & 87 \\
6 & $p-\mathrm{CH}_{3} \mathrm{C}_{6} \mathrm{H}_{4}$ & $\mathrm{Br}$ & $\mathrm{NaOCH}_{3}$ & $\mathrm{CH}_{3} \mathrm{OH}$ & $\mathbf{7 f}$ & 90 \\
7 & $p-\mathrm{ClC}_{6} \mathrm{H}_{4}$ & $\mathrm{Br}$ & $\mathrm{NaOCH}_{3}$ & $\mathrm{CH}_{3} \mathrm{OH}$ & $\mathbf{7 g}$ & 80 \\
8 & $p-\mathrm{CH}_{3} \mathrm{OC}_{6} \mathrm{H}_{4}$ & $\mathrm{Br}$ & $\mathrm{NaOCH}_{3}$ & $\mathrm{CH}_{3} \mathrm{OH}$ & $\mathbf{7 h}$ & 82 \\
9 & $\mathrm{C}_{6} \mathrm{H}_{5}$ & $\mathrm{I}$ & $\mathrm{NaOC}_{2} \mathrm{H}_{5}$ & $\mathrm{C}_{2} \mathrm{H}_{5} \mathrm{OH}$ & $\mathbf{7 i}$ & 86 \\
10 & $p-\mathrm{CH}_{3} \mathrm{C}_{6} \mathrm{H}_{4}$ & $\mathrm{I}$ & $\mathrm{NaOC}_{2} \mathrm{H}_{5}$ & $\mathrm{C}_{2} \mathrm{H}_{5} \mathrm{OH}$ & $\mathbf{7 j}$ & 82 \\
11 & $\mathrm{C}_{6} \mathrm{H}_{5}$ & $\mathrm{Br}$ & $\mathrm{NaOC}_{2} \mathrm{H}_{5}$ & $\mathrm{C}_{2} \mathrm{H}_{5} \mathrm{OH}$ & $\mathbf{7 k}$ & 73 \\
12 & $p-\mathrm{CH}_{3} \mathrm{C}_{6} \mathrm{H}_{4}$ & $\mathrm{Br}$ & $\mathrm{NaOC}_{2} \mathrm{H}_{5}$ & $\mathrm{C}_{2} \mathrm{H}_{5} \mathrm{OH}$ & $\mathbf{7 l}$ & 81 \\
\hline
\end{tabular}

${ }^{a}$ Isolated yields after purification. ${ }^{b}$ Reaction time 90 minutes

The diastereomerically pure, functionalized alkyl 4-iodo-5-aryl-3-(arylamino)pyrrolidine-2carboxylates (7) thus obtained were characterized on the basis of analytical and spectral evidence. The compound, methyl 4-iodo-5-phenyl-3-(phenylamino)pyrrolidine-2-carboxylate 7a for example, analyzed for $\mathrm{C}_{18} \mathrm{H}_{19} \mathrm{IN}_{2} \mathrm{O}_{2}$ showed a $(\mathrm{M}+1)$ molecular ion peak at $\mathrm{m} / \mathrm{z}, 423$ in its mass spectrum (Figure 2). The ${ }^{1} \mathrm{H}$ NMR $(300 \mathrm{MHz})$ spectrum showed a characteristic doublet $(\mathrm{J} 7.5 \mathrm{~Hz})$ at $\delta 4.70$ corresponding to $\mathrm{H}_{2}$ of the ring, a broad singlet at $\delta 4.46$ corresponding to $\mathrm{H}_{3}$ and $\mathrm{H}_{4}$ of the ring, a doublet at $\delta 4.11$ having $J 7.2 \mathrm{~Hz}$ assigned to $\mathrm{H}_{5}$. The ${ }^{13} \mathrm{C}$ NMR have shown the presence 
of one carbonyl carbon at $\delta 172.1$ and four aliphatic carbons at $\delta 71.0,66.0,61.7 \& 29.3$ corresponding to $\mathrm{C}-5, \mathrm{C}-2, \mathrm{C}-4$ and $\mathrm{C}-3$ respectively.

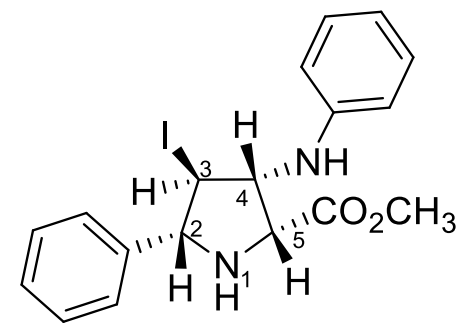

Figure 2. Methyl 4-iodo-5-phenyl-3-(phenylamino)pyrrolidine-2-carboxylate 7a.

4-Halo-5-phenyl-3-arylaminopyrrolidine-2-carboxylic acid alkyl esters 7 were also explored for the synthesis of 3,6-diazabicyclo[3.1.0]hexane-2-carboxylic acids $\mathbf{8}$ by intramolecular nucleophilic substitution reaction (90\%; Scheme-6). The intramolecular nucleophilic substitution reactions were studied at different temperature using different solvents to provide $N$-deprotected 4,6-diaryl-3,6-diazabicyclo[3.1.0]hexane-2-carboxylic acids 8 in good yields (Table-5, Entries 116) at $50^{\circ} \mathrm{C}$. We have also studied the one pot formation of $\mathbf{8}$ by the treatment of $\mathbf{2}$ with sodium alkoxide in corresponding alcohol at $50{ }^{\circ} \mathrm{C}$. From these observations, it may be concluded that there was initial formation of 7 at $50^{\circ} \mathrm{C}$ which underwent intramolecular nucleophilic substitution reaction to yield $\mathbf{8 a - d}$.

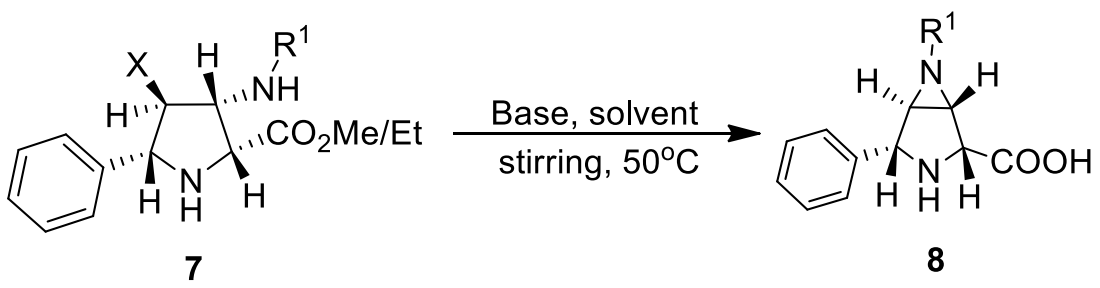

Scheme 6. Synthesis of 4,6-diaryl-3,6-diazabicyclo[3.1.0] hexane-2-carboxylic acids 8

The diastereomerically pure, functionalized novel 4,6-diaryl-3,6-diazabicyclo[3.1.0] hexane2-carboxylic acids 8 thus obtained were characterized on the basis of analytical and spectral evidence. (Figure 3) The compound, methyl 4,6-diphenyl-3,6-diazabicyclo[3.1.0]hexane-2carboxylic acid $\left(\mathrm{C}_{17} \mathrm{H}_{16} \mathrm{~N}_{2} \mathrm{O}_{2}\right)$ 8a for example, showed a molecular ion peak at $m / z(\mathrm{M}+1) 281$ in its mass spectrum. The ${ }^{1} \mathrm{H}$ NMR $(300 \mathrm{MHz})$ spectrum showed a characteristic doublet $(\mathrm{J} 1.8 \mathrm{~Hz})$ at $\delta 4.10$ corresponding to $\mathrm{H}_{2}$ of the ring, a doublet $(J 1.8 \mathrm{~Hz})$ at $\delta 3.68$ corresponding to $\mathrm{H}_{5}$ of the ring, two doublet of doublet at $\delta 3.23 \& 3.08$ having $J 4.5,2.1 \mathrm{~Hz}$ assigned to $\mathrm{H}_{3}$ and $\mathrm{H}_{4}$ respectively. The ${ }^{13} \mathrm{C}$ NMR have shown the presence of one carbonyl carbon at $\delta 173.8$ and four aliphatic carbons at $\delta 64.3,63.6,49.7,49.0$ corresponding to C-5, C-2, C-3 and C-4 respectively. 
Table 5. 4,6-diaryl-3,6-diazabicyclo[3.1.0] hexane-2-carboxylic acids 8

\begin{tabular}{ccccccc}
\hline S.No & $\mathrm{R}^{1}$ & $\mathrm{X}$ & Base & Solvent & Product & $\begin{array}{c}\text { Yield } \\
(\%)^{\mathrm{a}}\end{array}$ \\
\hline 1 & $\mathrm{C}_{6} \mathrm{H}_{5}$ & $\mathrm{I}$ & $\mathrm{NaOCH}_{3}$ & $\mathrm{CH}_{3} \mathrm{OH}$ & $\mathbf{8 a}$ & 90 \\
2 & $p-\mathrm{CH}_{3} \mathrm{C}_{6} \mathrm{H}_{4}$ & $\mathrm{I}$ & $\mathrm{NaOCH}_{3}$ & $\mathrm{CH}_{3} \mathrm{OH}$ & $\mathbf{8 b}$ & 88 \\
3 & $p-\mathrm{ClC}_{6} \mathrm{H}_{4}$ & $\mathrm{I}$ & $\mathrm{NaOCH}_{3}$ & $\mathrm{CH}_{3} \mathrm{OH}$ & $\mathbf{8 c}$ & 82 \\
4 & $p-\mathrm{CH}_{3} \mathrm{OC}_{6} \mathrm{H}_{4}$ & $\mathrm{I}$ & $\mathrm{NaOCH}_{3}$ & $\mathrm{CH}_{3} \mathrm{OH}$ & $\mathbf{8 d}$ & 85 \\
5 & $\mathrm{C}_{6} \mathrm{H}_{5}$ & $\mathrm{Br}$ & $\mathrm{NaOCH}_{3}$ & $\mathrm{CH}_{3} \mathrm{OH}$ & $\mathbf{8 a}$ & 87 \\
6 & $p-\mathrm{CH}_{3} \mathrm{C}_{6} \mathrm{H}_{4}$ & $\mathrm{Br}$ & $\mathrm{NaOCH}_{3}$ & $\mathrm{CH}_{3} \mathrm{OH}$ & $\mathbf{8 b}$ & 79 \\
7 & $p-\mathrm{ClC}_{6} \mathrm{H}_{4}$ & $\mathrm{Br}$ & $\mathrm{NaOCH}_{3}$ & $\mathrm{CH}_{3} \mathrm{OH}$ & $\mathbf{8 c}$ & 82 \\
8 & $p-\mathrm{CH}_{3} \mathrm{OC}_{6} \mathrm{H}_{4}$ & $\mathrm{Br}$ & $\mathrm{NaOCH}_{3}$ & $\mathrm{CH}_{3} \mathrm{OH}$ & $\mathbf{8 d}$ & 80 \\
9 & $\mathrm{C}_{6} \mathrm{H}_{5}$ & $\mathrm{I}$ & $\mathrm{NaOC}_{2} \mathrm{H}_{5}$ & $\mathrm{C}_{2} \mathrm{H}_{5} \mathrm{OH}$ & $\mathbf{8 a}$ & 84 \\
10 & $p-\mathrm{CH}_{3} \mathrm{C}_{6} \mathrm{H}_{4}$ & $\mathrm{I}$ & $\mathrm{NaOC}_{2} \mathrm{H}_{5}$ & $\mathrm{C}_{2} \mathrm{H}_{5} \mathrm{OH}$ & $\mathbf{8 b}$ & 78 \\
11 & $p-\mathrm{ClC}_{6} \mathrm{H}_{4}$ & $\mathrm{I}$ & $\mathrm{NaOC}_{2} \mathrm{H}_{5}$ & $\mathrm{C}_{2} \mathrm{H}_{5} \mathrm{OH}$ & $\mathbf{8 c}$ & 81 \\
12 & $p-\mathrm{CH}_{3} \mathrm{OC}_{6} \mathrm{H}_{4}$ & $\mathrm{I}$ & $\mathrm{NaOC}_{2} \mathrm{H}_{5}$ & $\mathrm{C}_{2} \mathrm{H}_{5} \mathrm{OH}$ & $\mathbf{8 d}$ & 76 \\
13 & $\mathrm{C}_{6} \mathrm{H}_{5}$ & $\mathrm{Br}$ & $\mathrm{NaOC}_{2} \mathrm{H}_{5}$ & $\mathrm{C}_{2} \mathrm{H}_{5} \mathrm{OH}$ & $\mathbf{8 a}$ & 85 \\
14 & $p-\mathrm{CH}_{3} \mathrm{C}_{6} \mathrm{H}_{4}$ & $\mathrm{Br}$ & $\mathrm{NaOC}_{2} \mathrm{H}_{5}$ & $\mathrm{C}_{2} \mathrm{H}_{5} \mathrm{OH}$ & $\mathbf{8 b}$ & 78 \\
15 & $p-\mathrm{ClC}_{6} \mathrm{H}_{4}$ & $\mathrm{Br}$ & $\mathrm{NaOC}_{2} \mathrm{H}_{5}$ & $\mathrm{C}_{2} \mathrm{H}_{5} \mathrm{OH}$ & $\mathbf{8 c}$ & 79 \\
16 & $p-\mathrm{CH}_{3} \mathrm{OC}_{6} \mathrm{H}_{4}$ & $\mathrm{Br}$ & $\mathrm{NaOC}_{2} \mathrm{H}_{5}$ & $\mathrm{C}_{2} \mathrm{H}_{5} \mathrm{OH}$ & $\mathbf{8 d}$ & 82 \\
\hline
\end{tabular}

${ }^{\mathrm{a}}$ Isolated yields after purification

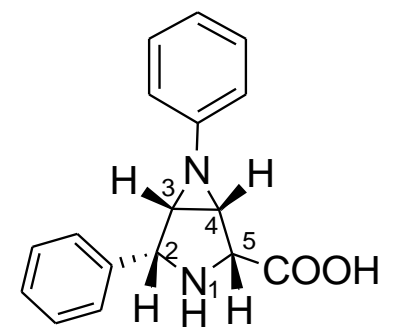

Figure 3. 4,6-Diphenyl-3,6-diazabicyclo[3.1.0]hexane-2-carboxylic acid 8a.

A plausible mechanism involves the initial formation of 4-halo-5-phenyl-3-arylaminopyrrolidine-2-carboxylate ester $\mathbf{7}$ as an intermediate in the transformation of 4-halo-3-phenyl-6aryl-2,6-diazabicyclo[3.2.0]heptan-7-one (2) into 4,6-diaryl-3,6-diazabicyclo[3.1.0]hexane-2carboxylic acid (8) (Scheme-7). The intramolecular nucleophilic attack of nitrogen in intermediate 9 to the adjacent halogenated carbon of pyrrole ring thereby yielding corresponding diazabicyclo[3.1.0]hexane-2-carboxylic acid in good yields. 

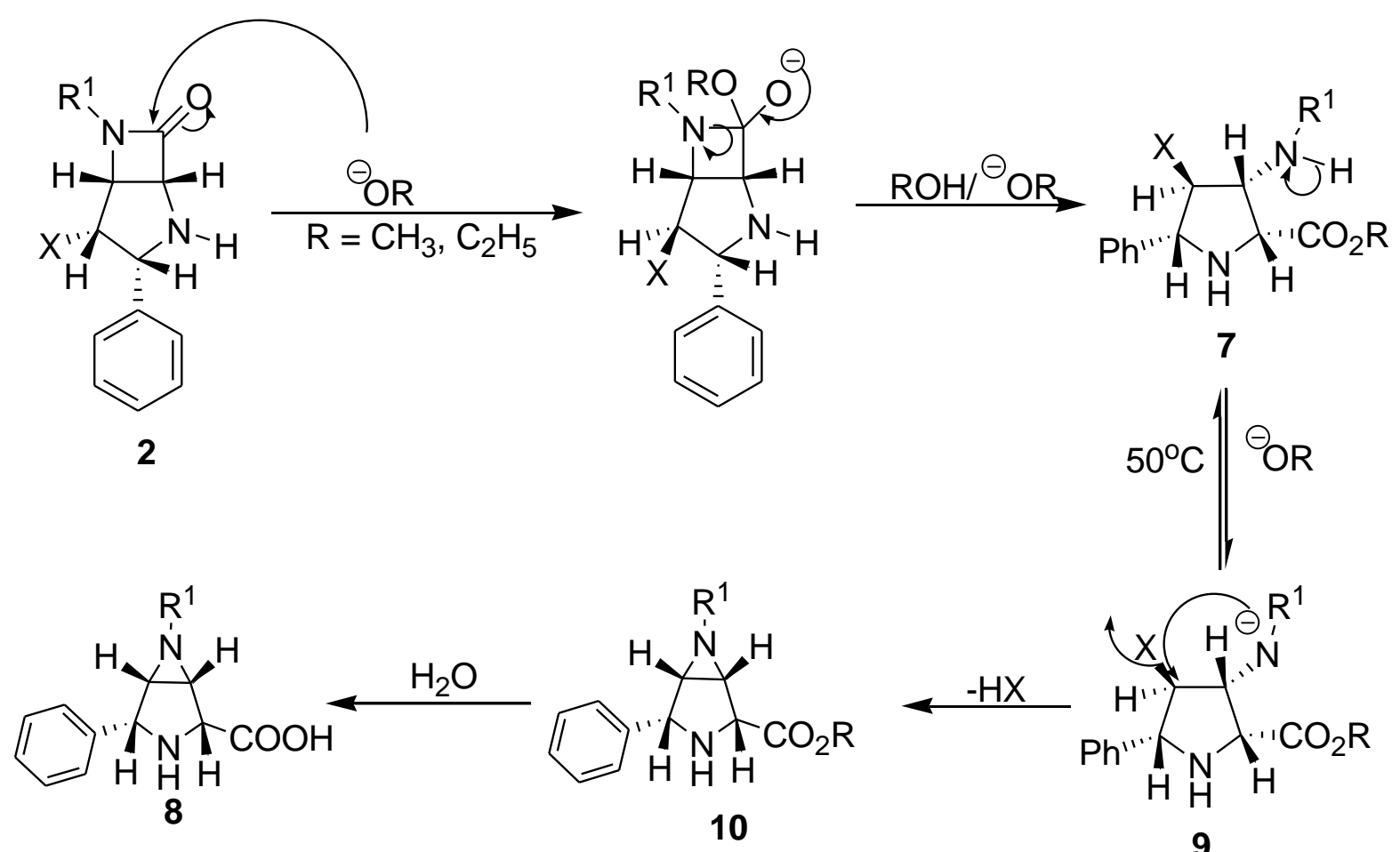

Scheme 7. Plausible mechanism depicting the formation of 4,6-diaryl-3,6-diazabicyclo[3.1.0]hexane-2-carboxylic acids $\mathbf{8}$.

\section{Conclusions}

We have developed a simple, convenient and metal free diastereoselective method for the functionally decorated 4-halo-3,6-diaryl-2,6-diazabicyclo[3.2.0]heptan-7-ones via intramolecular endo-trig haloamination of 3-aminoazetidin-2-ones in good to excellent yield and competitive exotrig haloamination was not observed. These diazabicyclo[3.2.0]heptan-7-ones served as novel $\beta$-lactam synthons for the synthesis of highly functionalized proline esters. The one pot amidiolytic ring opening of diazabicyclo[3.2.0] heptan-7-ones with sodium alkoxide also provided an easy access to previously unknown $N$-deprotected diazabicyclo[3.1.0]hexane-2-carboxylic acids in good yields.

\section{Experimental Section}

General. Oxygen- and moisture-sensitive reactions were carried out under nitrogen atmosphere. Solvents were purified and dried by standard methods prior to use. All commercially available reagents and solvents (purchased from Aldrich, Merck, Spectrochem, Acros) were used without further purification unless otherwise noted. Analytical thin layer chromatography (TLC) was 
conducted on Merck Kieselgel 60 F254. Compounds were visualized with both short- and longwavelength UV light. Column chromatography was performed on silica gel (100-200 mesh). Melting points were determined in capillary tubes using a Mel-Temp apparatus and are not corrected. Infrared spectra were obtained as films on $\mathrm{KBr}$ salt plates except where otherwise specified, using a Perkin Elmer FT-IR spectrometer. ${ }^{1} \mathrm{H}$ NMR spectra were obtained with $\mathrm{CDCl}_{3}$ at $300 \& 500 \mathrm{MHz}$, using Bruker spectrometers (residual chloroform referenced to $7.26 \mathrm{ppm}$ ) or DMSO- $d_{6}$ (residual DMSO referenced to $2.50 \mathrm{ppm}$ and residual water in DMSO- $d_{6}$ appearing at $3.33 \mathrm{ppm}$ ). Chemical shift values are expressed as parts per million downfield from TMS and $J$ values are in hertz. Splitting patterns are indicated as s: singlet, d: doublet, t: triplet, m: multiplet, dd: double doublet, ddd: doublet of a doublet of a doublet, and br: broad peak. ${ }^{13} \mathrm{C}$ NMR spectra were recorded with $\mathrm{CDCl}_{3}$ at $75 \mathrm{MHz}$, using Bruker spectrometers (residual chloroform referenced to $77.0 \mathrm{ppm}$ ) or DMSO- $d_{6}$ (residual DMSO referenced to $39.5 \mathrm{ppm}$ ). Infrared spectra were recorded on a Perkin Elmer FT-IR spectrometer. HRMS were recorded on Bruker high resolution spectrometer (Bruker microTOF QII).

General procedure for synthesis of compound 4-halo-3,6-diaryl-2,6-diazabicyclo[3.2.0]heptan-7-one 2. To a solution of compounds 1 ( $0.1 \mathrm{~g}, 1$ equiv) in DCM (10 $\mathrm{mL}$ ) was added bromine/iodine ( 1.2 equiv). The reaction was stirred for 10 minutes. This was followed by addition of $\mathrm{K}_{2} \mathrm{CO}_{3}$ at $0{ }^{\circ} \mathrm{C}$. The solution was stirred at $0{ }^{\circ} \mathrm{C}$ for $1-2 \mathrm{~h}$. The progress of the reaction was monitored with the help of tlc. After completion of the reaction, reaction mixture was diluted with DCM and washed with $\mathrm{Na}_{2} \mathrm{~S}_{2} \mathrm{O}_{3}$ /water solution followed by brine solution. The dichloromethane solution was dried over anhydrous $\mathrm{Na}_{2} \mathrm{SO}_{4}$ and solvent was evaporated. Crude residue was purified by flash column chromatography using silica gel (100:200 mesh) in EtOAc/cyclohexane (2:8) as an elutent system to get compounds 2 .

4-Iodo-3,6-diphenyl-2,6-diazabicyclo[3.2.0]heptan-7-one (2a). Yield: 90\%; White solid, mp 118-119 ${ }^{\circ} \mathrm{C} ; \delta_{\mathrm{H}}{ }^{1} \mathrm{H}$ NMR $\left(300 \mathrm{MHz}, \mathrm{CDCl}_{3}\right) 7.36$ (d, J 7.2 Hz, 2H), 7.10-7.19 (m 5H), 6.97$7.04(\mathrm{t}, J 7.5 \mathrm{~Hz}, 1 \mathrm{H}), 6.90(\mathrm{~d}, J 7.5 \mathrm{~Hz}, 2 \mathrm{H}), 5.02$ (d, J $3.9 \mathrm{~Hz}, 2 \mathrm{H}), 4.94$ (bs, 1H), 4.91 (d, J 3.6 $\mathrm{Hz}, 1 \mathrm{H}) . \delta_{\mathrm{C}} \mathrm{NMR}\left(75 \mathrm{MHz}, \mathrm{CDCl}_{3}\right) \delta 164.2,139.6,136.1,129.0,128.2,127.2,125.3,124.4$, 116.8, 74.7, 71.8, 67.8, 30.7. MS (EI) $\mathrm{m} / z .391(\mathrm{M}+1)^{+}, v_{\max }(\mathrm{KBr}) / \mathrm{cm}^{-1} 1755$, HRMS calculated for $\mathrm{C}_{17} \mathrm{H}_{15} \mathrm{IN}_{2} \mathrm{O}(\mathrm{M}+\mathrm{H})^{+}$391.0307, found 391.0314.

X-Ray crystal data and structure refinement. CCDC 972460 contains the supplementary crystallographic data. $\mathrm{C}_{17} \mathrm{H}_{15} \mathrm{I}_{1} \mathrm{~N}_{2} \mathrm{O}_{1}, \mathrm{~V}=2958.9(2) \AA^{3} \mathrm{Mr}=390.21, \mathrm{Z}=8$, orthorhombic, $\mathrm{a}=$ $9.8710(5) \AA, m=2.165 \mathrm{~mm}^{-1}, \mathrm{~b}=16.0822(8) \AA, \mathrm{T}=100(2) \mathrm{K}, \mathrm{c}=18.6387(8) \AA, a=90, b=90$ $g=90 ; \quad \mathrm{b}=104.719(2), \mathrm{T}_{\min }=0.655, \mathrm{~T}_{\max }=0.677, \mathrm{R}_{\mathrm{int}}=0.0252,3047$ measured reflections, $\mathrm{wR}(\mathrm{F} 2)=0.0759, \mathrm{~S}=1.155$

4-Iodo-3-phenyl-6-(p-tolyl)-2,6-diazabicyclo[3.2.0]heptan-7-one (2b). Yield: 82\%; White solid, mp $129-131^{\circ} \mathrm{C} ; \delta_{\mathrm{H}}{ }^{1} \mathrm{H}$ NMR $\left(300 \mathrm{MHz}, \mathrm{CDCl}_{3}\right) 7.37(\mathrm{dd}, J 6.9,0.9 \mathrm{~Hz}, 2 \mathrm{H})$, $7.11-7.21(\mathrm{~m}, 3 \mathrm{H}), 6.96(\mathrm{~d}, J 8.1 \mathrm{~Hz}, 1 \mathrm{H}), 6.78(\mathrm{dd}, J 6.6,1.8 \mathrm{~Hz}, 2 \mathrm{H}), 5.01(\mathrm{~d}, J 3.6 \mathrm{~Hz}$, $2 \mathrm{H}), 4.91(\mathrm{bs}, 2 \mathrm{H}), 2.24(\mathrm{~s}, 3 \mathrm{H}) . \delta_{\mathrm{C}} \mathrm{NMR}\left(75 \mathrm{MHz}, \mathrm{CDCl}_{3}\right) \delta 163.9,139.7,134.2,133.6$, 
$129.5,128.2,127.1,125.4,116.8,74.8,71.8,67.8,30.8,20.9$. MS (EI) $m / z 405(\mathrm{M}+1)^{+}, v_{\max }$ $(\mathrm{KBr}) / \mathrm{cm}^{-1} 1755$, HRMS calculated for $\mathrm{C}_{18} \mathrm{H}_{17} \mathrm{IN}_{2} \mathrm{O}(\mathrm{M}+\mathrm{H})^{+} 405.0464$, found 405.0488. 6-(4-Chlorophenyl)-4-iodo-3-phenyl-2,6-diazabicycle[3.2.0]heptan-7-one (2c). Yield: 65\%; Pale yellow solid, mp 143-144; $\delta_{\mathrm{H}}{ }^{1} \mathrm{H}$ NMR $\left(300 \mathrm{MHz}, \mathrm{CDCl}_{3}\right) 7.35$ (dd, J 8.1, 1.2 $\mathrm{Hz}, 2 \mathrm{H}), 7.10-7.20(\mathrm{~m}, 5 \mathrm{H}), 6.83(\mathrm{~d}, J 6.6 \mathrm{~Hz}, 2 \mathrm{H}), 5.01$ (d, J 3.6 Hz, 2H), 4.92 (bs, 2H). $\delta_{\mathrm{C}} \mathrm{NMR}\left(75 \mathrm{MHz}, \mathrm{CDCl}_{3}\right) \delta 164.1,139.5,134.6,129.1,128.9,128.3,127.2,125.3,118.0$, 74.5, 72.2, 67.9, 30.3. MS (EI) $\mathrm{m} / z 425(\mathrm{M}+1)^{+}, v_{\max }(\mathrm{KBr}) / \mathrm{cm}^{-1} 1755$, HRMS calculated for $\mathrm{C}_{17} \mathrm{H}_{14} \mathrm{ClIN}_{2} \mathrm{O}(\mathrm{M}+\mathrm{H})^{+} 424.9918$, found 424.9915 .

\section{4-Iodo-6-(4-methoxyphenyl)-3-phenyl-2,6-diazabicyclo[3.2.0]heptan-7-one}

(2d). Yield: 66\%; White solid, mp 137-139; $\delta_{\mathrm{H}}{ }^{1} \mathrm{H}$ NMR $\left(300 \mathrm{MHz}, \mathrm{CDCl}_{3}\right) 7.37$ (d, J $7.2 \mathrm{~Hz}$, 2H), 7.10-7.19 (m, 5H), 6.80 (dd, J 6.6, $1.8 \mathrm{~Hz}, 2 \mathrm{H}), 5.01$ (d, J 3.6 Hz, 2H ), 4.91 (bs, 2H), $3.21(\mathrm{~s}, 3 \mathrm{H}) . \delta_{\mathrm{C}} \mathrm{NMR}\left(75 \mathrm{MHz}, \mathrm{CDCl}_{3}\right) \delta 164.0,139.7,134.2,133.7,129.5,128.2,127.1$, 125.3, 116.8, 74.8, 71.7, 67.8, 55.9, 30.8. MS (EI) $\mathrm{m} / \mathrm{z} 421(\mathrm{M}+1)^{+}, v_{\max }(\mathrm{KBr}) / \mathrm{cm}^{-1} 1755$, HRMS calculated for $\mathrm{C}_{18} \mathrm{H}_{17} \mathrm{IN}_{2} \mathrm{O}_{2}(\mathrm{M}+\mathrm{H})^{+} 421.0413$, found 421.0411 .

6-(4-Fluorophenyl)-4-iodo-3-phenyl-2,6-diazabicycle[3.2.0]heptan-7-one (2e). Yield: 62\%; Pale yellow solid, mp 124-127; $\delta_{\mathrm{H}}{ }^{1} \mathrm{H}$ NMR $\left(300 \mathrm{MHz}, \mathrm{CDCl}_{3}\right)$ 7.34-7.37 (m, 2H, ArH), 7.10-7.26 (m, 5H, ArH), 6.82-6.85 (m, 2H, ArH), 5.00 (d, J 3.6 Hz, 2H, H \& H4), $4.92\left(\mathrm{~m}, 2 \mathrm{H}, \mathrm{H}_{1} \& \mathrm{H}_{5}\right) . \delta_{\mathrm{C}} \mathrm{NMR}\left(75 \mathrm{MHz}, \mathrm{CDCl}_{3}\right) \delta 163.9,139.7,134.2,133.7,129.6$, $128.3,128.0,127.2,125.4,116.9,74.8,71.8,67.9,30.8$. MS (EI) $m / z 409(\mathrm{M}+1)^{+}, v_{\max }$ $(\mathrm{KBr}) / \mathrm{cm}^{-1} 1750$, HRMS calculated $(\mathrm{M}+\mathrm{H})^{+} 409.0213$, found 409.0207, Anal. Calc. for $\mathrm{C}_{17} \mathrm{H}_{14} \mathrm{FIN}_{2} \mathrm{O}$ : C, 50.02; H, 3.46; N, 6.86; found: C, 50.06; H, 3.51; N, 6.81.

6-Cyclohexyl-4-iodo-3-phenyl-2,6-diazabicyclo[3.2.0]heptan-7-one (2f). Yield: 75\%; Pale yellow solid, mp 110-111; $\delta_{\mathrm{H}}{ }^{1} \mathrm{H}$ NMR (500 MHz, $\left.\mathrm{CDCl}_{3}\right)$ 7.18-7.39 (m, 5H, ArH), $5.07\left(\mathrm{~d}, J 4.0 \mathrm{~Hz}, 2 \mathrm{H}, \mathrm{H}_{3} \& \mathrm{H}_{4}\right), 5.02\left(\mathrm{~s}, 1 \mathrm{H}, \mathrm{H}_{1}\right) 5.00\left(\mathrm{~d}, J 3.5 \mathrm{~Hz}, 1 \mathrm{H}, \mathrm{H}_{5}\right), 3.57-3.62(\mathrm{~m}$, $1 \mathrm{H}$, cyclohexyl-H), 0.85-1.95(m, 10H, cyclohexyl-H), $\delta_{\mathrm{C}} \mathrm{NMR}\left(75 \mathrm{MHz}, \mathrm{CDCl}_{3}\right) \delta 164.5$, 128.8, 128.7, 126.7 123.6, 74.7, 71.8, 67.9, 52.7, 31.8, 30.6, 29.7, 25.0. MS (EI) $\mathrm{m} / z 397$ $(\mathrm{M}+1)^{+}, v_{\max }(\mathrm{KBr}) / \mathrm{cm}^{-1}$ 1755, HRMS calculated $(\mathrm{M}+\mathrm{H})^{+}$397.0777, found 397.0773, Anal. Calc. for $\mathrm{C}_{17} \mathrm{H}_{21} \mathrm{IN}_{2} \mathrm{O}: \mathrm{C}, 51.53 ; \mathrm{H}, 5.34 ; \mathrm{N}, 7.07$; found: C, 51.60; H, 5.39; N, 7.04.

6-Benzyl-4-iodo-3-phenyl-2,6-diazabicyclo[3.2.0]heptan-7-one (2g). Yield: 60\%; Yellow solid, mp 125-126; $\delta_{\mathrm{H}}{ }^{1} \mathrm{H}$ NMR $\left(500 \mathrm{MHz}, \mathrm{CDCl}_{3}\right)$ 7.21-7.36 (m, 10H, ArH), 5.04 (d, J $\left.3.5 \mathrm{~Hz}, 2 \mathrm{H}, \mathrm{H}_{3} \& \mathrm{H}_{4}\right), 4.94\left(\mathrm{~s}, 1 \mathrm{H}, \mathrm{H}_{1}\right) 4.92$ (d, J 3.5 Hz, 1H, H5), 4.09-4.14 (m, 2H, $\left.\mathrm{CH}_{2}\right) . \delta_{\mathrm{C}} \mathrm{NMR}\left(75 \mathrm{MHz}, \mathrm{CDCl}_{3}\right) \delta 170.1,143.4,128.8,128.7,128.5,127.9,127.2,126.6$, 123.5, 74.4, 70.3, 65.8, 47.26, 29.7. MS (EI) $\mathrm{m} / z 405(\mathrm{M}+1)^{+}, v_{\max }(\mathrm{KBr}) / \mathrm{cm}^{-1} 1752$, HRMS calculated $(\mathrm{M}+\mathrm{H})^{+}$405.0464, found 405.0462, Anal. Calc. for $\mathrm{C}_{18} \mathrm{H}_{17} \mathrm{IN}_{2} \mathrm{O}: \mathrm{C}, 53.48 ; \mathrm{H}$, 4.24; N, 6.93; found: C, 53.52; H, 4.29; N, 6.89.

4-Bromo-3,6-diphenyl-2,6-diazabicyclo[3.2.0]heptan-7-one (2h). Yield: 61\%; Brown solid, mp 131-132; $\delta_{\mathrm{H}}{ }^{1} \mathrm{H}$ NMR $\left(300 \mathrm{MHz} \mathrm{CDCl}_{3}\right) 7.38(\mathrm{~m}, 2 \mathrm{H}, \mathrm{ArH}), 7.10-7.22$ (m, 5H, ArH), 6.97-7.04 (m, 1H, ArH), 6.93 (m, 2H, ArH), 5.02 (bs, 1H, H3), 4.92 (d, J 3.6 Hz, 1H, $\left.\left.\mathrm{H}_{4}\right), 4.91\left(\mathrm{bs}, 1 \mathrm{H}, \mathrm{H}_{1}\right), 4.83\left(\mathrm{~d}, J 3.6 \mathrm{~Hz}, 1 \mathrm{H}, \mathrm{H}_{5}\right) . \delta \mathrm{C} \mathrm{NMR} 75 \mathrm{MHz}, \mathrm{CDCl}_{3}\right) \delta 164.1,139.0$, 136.1, 129, 128.2, 127.2, 125.4, 124.5, 116.7, 73.1, 71.7, 66.1, 52.7. MS (EI) $\mathrm{m} / z 343$ 
$(\mathrm{M}+1)^{+}$, Anal. Calc. for $\mathrm{C}_{17} \mathrm{H}_{15} \mathrm{BrN}_{2} \mathrm{O}$ : C, 59.49; H, 4.41; N, 8.16; found: C, 59.41; H, 4.38; N, 8.20.

4-Bromo-3-phenyl-6-(p-tolyl)-2,6-diazabicyclo[3.2.0]heptan-7-one (2i). Yield: 55\%; Brown solid; $\delta_{\mathrm{H}}{ }^{1} \mathrm{H}$ NMR $\left(300 \mathrm{MHz}, \mathrm{CDCl}_{3}\right)$ 7.39(m, 2H, ArH), 7.10-7.23 (m, 3H, ArH), $6.97(\mathrm{~m}, 2 \mathrm{H}, \mathrm{ArH}), 6.80(\mathrm{~m}, 2 \mathrm{H}, \mathrm{ArH}), 5.02\left(\mathrm{~s}, 1 \mathrm{H}, \mathrm{H}_{3}\right), 4.91$ (d, J $\left.3.6 \mathrm{~Hz}, 1 \mathrm{H}, \mathrm{H}_{4}\right), 4.90$ (bs, $\left.1 \mathrm{H}, \mathrm{H}_{1}\right), 4.81\left(\mathrm{~d}, J 3.6 \mathrm{~Hz}, 1 \mathrm{H}, \mathrm{H}_{5}\right), 2.24\left(\mathrm{~s}, 3 \mathrm{H}, \mathrm{CH}_{3}\right) . \delta_{\mathrm{C}} \mathrm{NMR}\left(75 \mathrm{MHz}, \mathrm{CDCl}_{3}\right) \delta$ 163.8, 139.0, 134.2, 133.6, 129.5, 128.2, 127.2, 125.4, 116.8, 73.1, 71.6, 66.2, 52.7, 20.9. MS (EI) $m / z 357(\mathrm{M}+1)^{+}$, Anal. Calc. for $\mathrm{C}_{18} \mathrm{H}_{17} \mathrm{BrN}_{2} \mathrm{O}: \mathrm{C}, 60.52 ; \mathrm{H}, 4.80 ; \mathrm{N}, 7.84$; found: C, 60.49; H, 4.75; N, 7.87.

4-Bromo-6-(4-chlorophenyl)-3-phenyl-2,6-diazabicyclo[3.2.0]heptan-7-one

(2j).

Yield: 50\%; Light brown solid, $\delta_{\mathrm{H}}{ }^{1} \mathrm{H}$ NMR (300 MHz, $\left.\mathrm{CDCl}_{3}\right)$ 7.30-7.40 (m, 3H, ArH), 7.16-7.22 (m, 2H), $7.13(\mathrm{~m}, 2 \mathrm{H}, \mathrm{ArH}), 6.86(\mathrm{~m}, 2 \mathrm{H}, \mathrm{ArH}), 5.02\left(\mathrm{~s}, 1 \mathrm{H}, \mathrm{H}_{3}\right), 4.93$ (d, J 3.6 $\left.\mathrm{Hz}, 1 \mathrm{H}, \mathrm{H}_{4}\right), 4.89\left(\mathrm{~s}, 1 \mathrm{H}, \mathrm{H}_{1}\right), 4.81\left(\mathrm{~d}, J 3.6 \mathrm{~Hz}, 1 \mathrm{H}, \mathrm{H}_{5}\right) . \delta_{\mathrm{C}} \mathrm{NMR}\left(75 \mathrm{MHz}, \mathrm{CDCl}_{3}\right) \delta$ $163.5,134.5,129.6,129.1,128.8,128.3,127.5,125.5,117.9,73.0,71.5,66.0,51.6 . \mathrm{MS}$ (EI) $\mathrm{m} / z 377(\mathrm{M}+1)^{+}$, Anal. Calc. for $\mathrm{C}_{17} \mathrm{H}_{14} \mathrm{BrClN}_{2} \mathrm{O}$ : C, 54.06; H, 3.74; N, 7.42; found: C, 54.03; H, 3.68; N, 7.45.

4-Bromo-6-(4-methoxyphenyl)-3-phenyl-2,6-diazabicyclo[3.2.0]heptan-7-one (2k). Yield: 55\%; Brown solid, $\delta_{\mathrm{H}}{ }^{1} \mathrm{H}$ NMR $\left(300 \mathrm{MHz}, \mathrm{CDCl}_{3}\right)$ 7.37-7.51 (m, 4H, ArH), 7.10$7.18(\mathrm{~m}, 2 \mathrm{H}, \mathrm{ArH}), 7.06$ (m, 2H, ArH), $6.86(\mathrm{~m}, 2 \mathrm{H}, \mathrm{ArH}), 5.01\left(\mathrm{~s}, 1 \mathrm{H}, \mathrm{H}_{3}\right), 4.92(\mathrm{~d}, J 3.6$ $\left.\mathrm{Hz}, 1 \mathrm{H}, \mathrm{H}_{4}\right), 4.91\left(\mathrm{~s}, 1 \mathrm{H}, \mathrm{H}_{1}\right), 4.83\left(\mathrm{~d}, J 3.6 \mathrm{~Hz}, 1 \mathrm{H}, \mathrm{H}_{5}\right), 3.18\left(\mathrm{~s}, 3 \mathrm{H}, \mathrm{OCH}_{3}\right) . \delta_{\mathrm{C}} \mathrm{NMR}(75$ $\left.\mathrm{MHz} \mathrm{CDCl}_{3}\right) \delta 164.0,134.3,129.7,129.2,128.8,128.3,127.5,125.4,116.8,73.1,71.6$, 66.2, 57.8, 52.7. MS (EI) $m / z 373(\mathrm{M}+1)^{+}$, Anal. Calc. for $\mathrm{C}_{18} \mathrm{H}_{17} \mathrm{BrN}_{2} \mathrm{O}_{2}$ : C, 57.92; H, 4.59; N, 7.51; found: C, 57.91; H, 4.55; N, 7.57.

4-Bromo-6-(4-fluorophenyl)-3-phenyl-2,6-diazabicyclo[3.2.0]heptan-7-one (2l). Yield: 60\%; Brown solid, $\delta_{\mathrm{H}}{ }^{1} \mathrm{H}$ NMR $\left(300 \mathrm{MHz}, \mathrm{CDCl}_{3}\right)$ 7.02-7.29 (m, 7H, ArH), 6.78-6.81 (m, $2 \mathrm{H}, \mathrm{ArH}), 4.76-4.96\left(\mathrm{~m}, 2 \mathrm{H}, \mathrm{H}_{3} \& \mathrm{H}_{4}\right), 4.66\left(\mathrm{t}, J 3.3 \mathrm{~Hz}, 1 \mathrm{H}, \mathrm{H}_{5}\right), 4.66\left(\mathrm{~s}, 1 \mathrm{H}, \mathrm{H}_{1}\right) . \delta_{\mathrm{C}} \mathrm{NMR}$ $\left(75 \mathrm{MHz} \mathrm{CDCl}_{3}\right) \delta 163.5,134.5,129.6,129.1,128.8,128.3,127.5,125.5,117.9,73.0$, 71.5, 66.0, 51.6. MS (EI) $\mathrm{m} / z 361(\mathrm{M}+1)^{+}$, Anal. Calc. for $\mathrm{C}_{17} \mathrm{H}_{14} \mathrm{FBrN}_{2} \mathrm{O}$ : C, 56.53; $\mathrm{H}$, 3.91 ; N, 7.76; found: C, 56.55; H, 3.96; N, 7.73 .

6-Benzyl-4-bromo-3-phenyl-2,6-diazabicyclo[3.2.0]heptan-7-one (2m). Yield: 45\%; Yellow solid, $\delta_{\mathrm{H}}{ }^{1} \mathrm{H}$ NMR $\left(500 \mathrm{MHz}, \mathrm{CDCl}_{3}\right)$ 7.23-7.37 (m, 10H, ArH), $5.01\left(\mathrm{~s}, 1 \mathrm{H}, \mathrm{H}_{3}\right)$, $4.93\left(\mathrm{~d}, J 3.5 \mathrm{~Hz}, 1 \mathrm{H}, \mathrm{H}_{4}\right), 4.81\left(\mathrm{~s}, 1 \mathrm{H}, \mathrm{H}_{1}\right), 4.68\left(\mathrm{~d}, J 3.5 \mathrm{~Hz}, 1 \mathrm{H}, \mathrm{H}_{5}\right), 4.10-4.15(\mathrm{~m}, 2 \mathrm{H}$, $\left.\mathrm{CH}_{2}\right) . \delta_{\mathrm{C}} \mathrm{NMR}\left(75 \mathrm{MHz}, \mathrm{CDCl}_{3}\right) \delta 169.1,143.4,128.8,128.7,128.6,127.8,127.2,126.7$, 123.5, 73.0, 69.9, 65.8, 50.6, 47.2. MS (EI) $m / z 357(\mathrm{M}+1)^{+}$, Anal. Calc. for $\mathrm{C}_{18} \mathrm{H}_{17} \mathrm{IN}_{2} \mathrm{O}$ : C, 60.52; H, 4.80; N, 7.84; found: C, 60.54; H, 4.85; N, 7.80.

General procedure for synthesis of 4-halo-2-alkyl-3,6-diaryl-2,6-diazabicyclo[3.2.0]heptan7-ones (6). To a solution of compounds 5 ( $0.1 \mathrm{~g}, 1$ equiv) in DCM (10 mL) was added bromine/iodine ( 1.2 equiv). The reaction was stirred for 10 minutes. This was followed by addition of $\mathrm{K}_{2} \mathrm{CO}_{3}$ at $0{ }^{\circ} \mathrm{C}$. The solution was stirred at $0{ }^{\circ} \mathrm{C}$. The progress of the reaction 
was monitored with the help of tlc. After completion of the reaction, reaction mixture was diluted with DCM and washed with $\mathrm{Na}_{2} \mathrm{~S}_{2} \mathrm{O}_{3} /$ water solution followed by brine solution. The dichloromethane solution was dried over anhydrous $\mathrm{Na}_{2} \mathrm{SO}_{4}$ and solvent was evaporated. Crude residue was purified by flash column chromatography using silica gel (100:200 mesh) in EtOAc/cyclohexane (2:8) as an elutent system to get compounds 6.

3-(Methylamino)-1-phenyl-4-((E)-styryl)azetidin-2-one (5a). White solid, $\delta_{\mathrm{H}}{ }^{1} \mathrm{H}$ NMR $\left(500 \mathrm{MHz} \mathrm{CDCl}_{3}\right) 7.48$ (m, 2H, ArH), 7.43-7.45 (m, 2H, ArH), 7.28-7.38 (m, 5H, ArH), $7.10(\mathrm{~m}, 1 \mathrm{H}, \mathrm{ArH}), 6.85\left(\mathrm{~d}, J 16.5 \mathrm{~Hz}, 1 \mathrm{H}, \mathrm{H}_{6}\right), 6.53\left(\mathrm{dd}, J 16.0,8.0 \mathrm{~Hz}, 1 \mathrm{H}, \mathrm{H}_{5}\right), 4.86(\mathrm{t}, J$ $\left.6.5 \mathrm{~Hz}, 1 \mathrm{H}, \mathrm{H}_{3}\right), 4.46\left(\mathrm{~d}, J 5.5 \mathrm{~Hz}, 1 \mathrm{H}, \mathrm{H}_{3}\right), 2.90\left(\mathrm{~s}, 3 \mathrm{H}, \mathrm{NCH}_{3}\right) . \delta_{\mathrm{C}} \mathrm{NMR}\left(75 \mathrm{MHz}, \mathrm{CDCl}_{3}\right)$ $\delta 165.9,138.3,136.0,135.3,129.1,128.3,128.1,126.6,124.3,116.7,57.5,56.1,32.4 . \mathrm{MS}$ (EI) $m / z 279(\mathrm{M}+1)^{+}$, Anal. Calc. for $\mathrm{C}_{18} \mathrm{H}_{18} \mathrm{~N}_{2} \mathrm{O}$ : C, 77.67; H, 6.52; N, 10.06; found: C, $77.71 ; \mathrm{H}, 6.54 ; \mathrm{N}, 10.02$.

3-(Dimethylamino)-1-phenyl-4-((E)-styryl)azetidin-2-one (5b). White solid, $\delta_{\mathrm{H}}{ }^{1} \mathrm{H}$ NMR (500 MHz, $\left.\mathrm{CDCl}_{3}\right)$ 7.46-7.51 (m, 4H, ArH), 7.28-7.40 (m, 5H, ArH), 7.07 (m, 1H, ArH), $6.75\left(\mathrm{~d}, J 16.0 \mathrm{~Hz}, 1 \mathrm{H}, \mathrm{H}_{6}\right), 6.30\left(\mathrm{dd}, J 15.5,9.0 \mathrm{~Hz}, 1 \mathrm{H}, \mathrm{H}_{5}\right), 4.88\left(\mathrm{t}, J 6.5 \mathrm{~Hz}, 1 \mathrm{H}, \mathrm{H}_{3}\right)$, $4.45\left(\mathrm{~d}, J 6.0 \mathrm{~Hz}, 1 \mathrm{H}, \mathrm{H}_{3}\right), 2.92\left(\mathrm{~s}, 6 \mathrm{H}, \mathrm{N}\left(\mathrm{CH}_{3}\right)_{2}\right) . \delta_{\mathrm{C}} \mathrm{NMR}\left(75 \mathrm{MHz}, \mathrm{CDCl}_{3}\right) \delta 165.9,138.7$, 136.6, 135.6, 130.1, 128.7, 128.1, 126.6, 124.6, 124.3, 116.3, 57.5, 56.8, 38.3. MS (EI) $\mathrm{m} / z$ $293(\mathrm{M}+1)^{+}$, Anal. Calc. for $\mathrm{C}_{19} \mathrm{H}_{20} \mathrm{~N}_{2} \mathrm{O}: \mathrm{C}, 78.05 ; \mathrm{H}, 6.89 ; \mathrm{N}, 9.58$; found: C, 78.11; H, $6.93 ; \mathrm{N}, 9.54$.

4-Methyl- $\boldsymbol{N}$-(2-oxo-1-phenyl-4-((E)-styryl)azetidin-3-yl)benzenesulfonamide (5c). White solid, $\delta_{\mathrm{H}}{ }^{1} \mathrm{H}$ NMR $\left(500 \mathrm{MHz}, \mathrm{CDCl}_{3}\right)$ 7.74-7.77 (m, 2H, ArH), 7.06-7.31 (m, 12H, ArH), 6.45 (d, J 15.5 Hz, 1H, H6), 5.93 (dd, J 16.0, 9.0 Hz, 1H, H5), 5.06 (t, J 6.5 Hz, 1H, $\left.\mathrm{H}_{3}\right), 4.78\left(\mathrm{~d}, J 6.5 \mathrm{~Hz}, 1 \mathrm{H}, \mathrm{H}_{3}\right), 2.71\left(\mathrm{~s}, 3 \mathrm{H}, \mathrm{CH}_{3}\right) . \delta_{\mathrm{C}} \mathrm{NMR}\left(75 \mathrm{MHz}, \mathrm{CDCl}_{3}\right) \delta 166.2$, 139.4, 137.0, 136.3, 136.0, 135.3, 131.5, 130.1, 129.4, 128.7, 128.1, 126.6, 124.6, 124.3, 117.1, 57.5, 56.1, 16.2. MS (EI) $m / z 419(\mathrm{M}+1)^{+}$, Anal. Calc. for $\mathrm{C}_{24} \mathrm{H}_{22} \mathrm{~N}_{2} \mathrm{O}_{3} \mathrm{~S}: \mathrm{C}, 68.88$; H, 5.30; N, 6.69; found: C, 68.95; H, 5.33; N, 6.65.

4-Iodo-2-methyl-3,6-diphenyl-2,6-diazabicyclo[3.2.0]heptan-7-one (6a). Yield: 75\%; White solid, $\delta_{\mathrm{H}}{ }^{1} \mathrm{H}$ NMR (500 MHz, $\left.\mathrm{CDCl}_{3}\right)$ 7.43-7.45 (m, 2H, ArH), 7.28-7.38 (m, 5H, ArH), 7.10 (m, 1H, ArH), 6.92 (m, 1H, ArH), 5.03 (d, J 4.0 Hz, 2H, H3 \& H4), 4.95 (s, 1H, $\left.\mathrm{H}_{1}\right), 4.92\left(\mathrm{~d}, J 3.0 \mathrm{~Hz}, 1 \mathrm{H}, \mathrm{H}_{5}\right), 2.40\left(\mathrm{~s}, 3 \mathrm{H}, \mathrm{CH}_{3}\right) . \delta \mathrm{C}$ NMR $\left(75 \mathrm{MHz}, \mathrm{CDCl}_{3}\right) \delta 163.9$, 139.4, 136.3, 129.4, 128.3, 127.4, 125.3, 124.6, 116.3, 74.7, 71.6, 67.5, 43.7, 30.7. MS (EI) $\mathrm{m} / \mathrm{z} 405(\mathrm{M}+1)^{+}$, HRMS calculated $(\mathrm{M}+\mathrm{H})^{+}$405.0464, found 405.0655, Anal. Calc. for $\mathrm{C}_{18} \mathrm{H}_{17} \mathrm{IN}_{2} \mathrm{O}$ : C, 53.48; H, 4.24; N, 6.93; found: C, 53.54; H, 4.30; N, 6.89.

4-Bromo-2-methyl-3,6-diphenyl-2,6-diazabicyclo[3.2.0]heptan-7-one (6b). Yield: 40\%; Brown solid; $\delta_{\mathrm{H}}{ }^{1} \mathrm{H}$ NMR $\left(500 \mathrm{MHz}, \mathrm{CDCl}_{3}\right)$ 7.43-7.49 (m, 4H, ArH), 7.28-7.37 (m, 5H, $\operatorname{ArH}), 7.10(\mathrm{~m}, 1 \mathrm{H}, \mathrm{ArH}), 5.00\left(\mathrm{~s}, 1 \mathrm{H}, \mathrm{H}_{3}\right), 4.81-4.89\left(\mathrm{~m}, 2 \mathrm{H}, \mathrm{H}_{1} \& \mathrm{H}_{4}\right), 4.82$ (d, J $3.5 \mathrm{~Hz}$, $\left.1 \mathrm{H}, \mathrm{H}_{5}\right), 2.44\left(\mathrm{~s}, 3 \mathrm{H}, \mathrm{CH}_{3}\right) . \delta_{\mathrm{C}} \mathrm{NMR}\left(75 \mathrm{MHz} \mathrm{CDCl}_{3}\right) \delta 165.3,138.0,135.6,129.1,128.7$, 
128.4, 126.7, 124.3, 117.0, 73.8, 71.9, 66.2, 52.7, 45.8. (EI) $m / z 357(\mathrm{M}+1)^{+}$, Anal. Calc. for $\mathrm{C}_{18} \mathrm{H}_{17} \mathrm{BrN}_{2} \mathrm{O}: \mathrm{C}, 60.52 ; \mathrm{H}, 4.80 ; \mathrm{N}, 7.84$; found: C, 60.50; H, 4.71; N, 7.78.

Typical procedure for the preparation of alkyl 4-iodo-5-aryl-3(arylamino)pyrrolidine-2-carboxylates (7). To a solution of compounds 2 (30mg, 1 eq) in methanol/ethanol $(5 \mathrm{~mL}), \mathrm{NaOMe} / \mathrm{NaOEt}$ ( $3 \mathrm{eq})$ was added and the reaction mixture was stirred at $0{ }^{\circ} \mathrm{C}$ for $1.5 \mathrm{~h}$. The progress of the reaction was monitored with the help of TLC. After completion of the reaction, the mixture was quenched with ice and $\mathrm{pH}$ adjust to 6-7 extracted with ethyl acetate (3 times). The combined organic layers were washed with water and brine, dried over anhydrous $\mathrm{Na}_{2} \mathrm{SO}_{4}$ and the solvent was evaporated to get compound (7) as a pure product as solid.

Methyl 4-iodo-5-phenyl-3-(phenylamino)pyrrolidine-2-carboxylate (7a). Yield: 85\%; White solid; $\delta_{\mathrm{H}}{ }^{1} \mathrm{H}$ NMR $\left(500 \mathrm{MHz}, \mathrm{CDCl}_{3}\right) 7.52(\mathrm{~m}, 2 \mathrm{H}, \mathrm{ArH}), 7.31-7.39$ (m, 3H, ArH), 7.19 (t, J 7.5 Hz, 2H, ArH), 6.76 (t, J 7.5 Hz, 1H, ArH), 6.63 (d, J 7.8 Hz, 2H, ArH), 4.70 $\left(\mathrm{d}, J 7.5 \mathrm{~Hz}, 1 \mathrm{H}, \mathrm{H}_{2}\right), 4.46\left(\mathrm{bs}, 2 \mathrm{H}, \mathrm{H}_{3} \& \mathrm{H}_{4}\right), 4.11\left(\mathrm{~d}, J 7.2 \mathrm{~Hz}, 1 \mathrm{H}, \mathrm{H}_{5}\right), 3.64(\mathrm{~s}, 3 \mathrm{H}$, $\left.\mathrm{COOCH}_{3}\right) . \delta \mathrm{C}$ NMR $\left(75 \mathrm{MHz}, \mathrm{CDCl}_{3}\right) \delta 172.1,145.8,139.3,129.3,128.9,128.4,127$, 118.7, 113.9, 71.0, 66.0, 61.7, 52.3, 29.3. MS (EI) $\mathrm{m} / z .423(\mathrm{M}+1)^{+}$, HRMS calculated $(\mathrm{M}+\mathrm{H})^{+}$423.0569, found 423.0561, Anal. Calc. for $\mathrm{C}_{18} \mathrm{H}_{19} \mathrm{IN}_{2} \mathrm{O}_{2}: \mathrm{C}, 51.20 ; \mathrm{H}, 4.54 ; \mathrm{N}$, 6.63; found: C, 51.12; H, 4.49; N, 6.69.

Methyl 4-iodo-5-phenyl-3-(p-tolylamino)pyrrolidine-2-carboxylate (7b). Yield: 88\%; White solid; $\delta_{\mathrm{H}}{ }^{1} \mathrm{H}$ NMR $\left(300 \mathrm{MHz}, \mathrm{CDCl}_{3}\right) 7.51(\mathrm{~m}, 2 \mathrm{H}, \mathrm{ArH}), 7.31-7.38$ (m, 3H, ArH), 6.99 (m, 2H, ArH), 6.53 (d, J 8.4 Hz, 2H, ArH), 4.69 (d, J 7.2 Hz, 1H, H2), 4.43 (bs, 2H, $\left.\mathrm{H}_{3} \& \mathrm{H}_{4}\right), 4.06-4.10\left(\mathrm{~m}, 1 \mathrm{H}, \mathrm{H}_{5}\right), 3.66\left(\mathrm{~s}, 3 \mathrm{H}, \mathrm{COOCH}_{3}\right), 2.23\left(\mathrm{~s}, 3 \mathrm{H}, \mathrm{CH}_{3}\right) . \delta \mathrm{C}$ NMR $(75$ $\left.\mathrm{MHz} \mathrm{CDCl}_{3}\right) \delta 172.3,143.5,140.1,129.6,128.7,128.2,126.9,120.3,114.1,71.3,66.5$, 61.8, 52.2, 33.9, 20.4. MS (EI) $m / z 437(\mathrm{M}+1)^{+}$, HRMS calculated $(\mathrm{M}+\mathrm{H})^{+} 437.0726$, found 437.0726, Anal. Calc. for $\mathrm{C}_{19} \mathrm{H}_{21} \mathrm{IN}_{2} \mathrm{O}_{2}$ : C, 52.31; H, 4.85; N, 6.42; found: C, 52.29; H, $4.80 ; \mathrm{N}, 6.44$.

Methyl 3-((4-chlorophenyl)amino)-4-iodo-5-phenylpyrrolidine-2-carboxylate (7c). Yield: 75\%; White solid; $\delta_{\mathrm{H}}{ }^{1} \mathrm{H}$ NMR $\left(500 \mathrm{MHz}, \mathrm{CDCl}_{3}\right)$ 7.43-7.45 (m, 2H, ArH), 7.287.39 (m, 3H, ArH), 7.12 (d, J 8.0 Hz, 2H, ArH), 6.81-6.89 (m, 2H, ArH), 4.69 (d, J 6.0 Hz, $\left.1 \mathrm{H}, \mathrm{H}_{2}\right), 4.48\left(\mathrm{bs}, 2 \mathrm{H}, \mathrm{H}_{3} \& \mathrm{H}_{4}\right), 4.12\left(\mathrm{~d}, J 7.0 \mathrm{~Hz}, 1 \mathrm{H}, \mathrm{H}_{5}\right), 3.63\left(\mathrm{~s}, 3 \mathrm{H}, \mathrm{CH}_{3}\right) . \delta_{\mathrm{C}} \mathrm{NMR}$ $\left(75 \mathrm{MHz} \mathrm{CDCl}_{3}\right) \delta 171.4,146.6,139.4,129.1,128.5,128.4,127.1,117.3,114.2,71.2$, 65.8, 61.8, 52.6, 29.7. MS (EI) $m / z 457(\mathrm{M}+1)^{+}$, HRMS calculated $(\mathrm{M}+\mathrm{H})^{+} 457.0180$, found 457.0177, Anal. Calc. for $\mathrm{C}_{18} \mathrm{H}_{18} \mathrm{ClIN}_{2} \mathrm{O}_{2}$ : C, 47.34; H, 3.97; N, 6.13; found: C, 47.31; H, $3.92 ; \mathrm{N}, 6.17$.

Methyl 4-iodo-3-((4-methoxyphenyl)amino)-5-phenylpyrrolidine-2-carboxylate (7d). Yield: 79\%; White solid; $\delta_{\mathrm{H}}{ }^{1} \mathrm{H}$ NMR $\left(500 \mathrm{MHz}, \mathrm{CDCl}_{3}\right)$ 7.32-7.38 (m, 4H, ArH), 7.107.25 (m, 3H, ArH), 6.83-6.87 (m, 2H, ArH), 4.69 (d, J 6.5 Hz, 1H, H $), 4.49$ (bs, 2H, H $\mathrm{H}_{3}$ \& $\left.\mathrm{H}_{4}\right), 4.15\left(\mathrm{~d}, J 7.5 \mathrm{~Hz}, 1 \mathrm{H}, \mathrm{H}_{5}\right), 3.77\left(\mathrm{~s}, 3 \mathrm{H}, \mathrm{OCH}_{3}\right), 3.60\left(\mathrm{~s}, 3 \mathrm{H}, \mathrm{COOCH}_{3}\right) . \delta_{\mathrm{C}} \mathrm{NMR}(75$ $\left.\mathrm{MHz} \mathrm{CDCl}_{3}\right) \delta 169.0,145.6,137.7,129.1,128.7,128.3,127.0,117.0,113.6,71.2,66.5$, 
61.3, 55.8, 52.0, 29.6. MS (EI) $\mathrm{m} / z 453(\mathrm{M}+1)^{+}$, HRMS calculated $(\mathrm{M}+\mathrm{H})$ 453.0675, found 453.0669, Anal. Calc. for $\mathrm{C}_{19} \mathrm{H}_{21} \mathrm{IN}_{2} \mathrm{O}_{3}$ : C, 50.46; H, 4.68; N, 6.19; found: C, 50.39; H, $4.63 ; \mathrm{N}, 6.21$.

Methyl 4-bromo-5-phenyl-3-(phenylamino)pyrrolidine-2-carboxylate (7e). Yield: 87\%; White solid; $\delta_{\mathrm{H}}{ }^{1} \mathrm{H}$ NMR $\left(300 \mathrm{MHz} \mathrm{CDCl}_{3}\right) 7.50(\mathrm{~m}, 2 \mathrm{H}, \mathrm{ArH}), 7.29-7.38$ (m, 3H, ArH), 7.17 (t, J 6.6 Hz, 2H, ArH), $6.75(\mathrm{t}, J 7.5 \mathrm{~Hz}, 1 \mathrm{H}, \operatorname{ArH}), 6.61$ (d, J 7.8 Hz, 2H, ArH), $4.60\left(\mathrm{~d}, J 6.0 \mathrm{~Hz}, 1 \mathrm{H}, \mathrm{H}_{2}\right), 4.48$ (d, J $\left.6 \mathrm{~Hz}, 1 \mathrm{H}, \mathrm{H}_{5}\right), 4.40$ (bs, 1H, H3), 4.07 (dd, J 3.9, 2.1 $\left.\mathrm{Hz}, 1 \mathrm{H}, \mathrm{H}_{4}\right), 3.68\left(\mathrm{~s}, 3 \mathrm{H}, \mathrm{COOCH}_{3}\right) . \delta_{\mathrm{C}} \mathrm{NMR}\left(75 \mathrm{MHz}, \mathrm{CDCl}_{3}\right) \delta 171.6,145.6,139.4$, $129.4,128.8,126.9,118.7,113.9,69.8,64.5,61.8,56.3,52.3,33.8$. MS (EI) $\mathrm{m} / z 375$ $(\mathrm{M}+1)^{+}$, HRMS calculated $(\mathrm{M}+\mathrm{H})^{+}$375.0708, found 375.0704, Anal. Calc. for $\mathrm{C}_{18} \mathrm{H}_{19} \mathrm{BrN}_{2} \mathrm{O}_{2}$ : C, 57.61; H, 5.10; N, 7.47; found: C, 57.59; H, 5.04; N, 7.50.

Methyl 4-bromo-5-phenyl-3-(p-tolylamino)pyrrolidine-2-carboxylate (7f). Yield: 90\%; White solid; $\delta_{\mathrm{H}}{ }^{1} \mathrm{H}$ NMR $\left(300 \mathrm{MHz}, \mathrm{CDCl}_{3}\right) 7.5(\mathrm{~m}, 2 \mathrm{H}, \mathrm{ArH}), 7.28-7.38(\mathrm{~m}, 3 \mathrm{H}$, ArH), 6.98 (d, J 7.8 Hz, 2H, ArH), 6.51 (d, J 7.8 Hz, 2H, ArH), 4.60 (d, J 5.7 Hz, 1H, H2), $4.47\left(\mathrm{~d}, J 5.7 \mathrm{~Hz}, 1 \mathrm{H}, \mathrm{H}_{5}\right), 4.36\left(\mathrm{bs}, 1 \mathrm{H}, \mathrm{H}_{3}\right), 4.06\left(\mathrm{dd}, J\right.$ 6, $\left.3.6 \mathrm{~Hz}, 1 \mathrm{H}, \mathrm{H}_{4}\right), 3.68(\mathrm{~s}, 3 \mathrm{H}$, $\left.\mathrm{COOCH}_{3}\right), 2.22\left(\mathrm{~s}, 3 \mathrm{H}, \mathrm{CH}_{3}\right) . \delta_{\mathrm{C}} \mathrm{NMR}\left(75 \mathrm{MHz}, \mathrm{CDCl}_{3}\right) \delta 171.8,143.3,139.9,129.8$, 128.8, 128.2, 128.0, 126.8, 114.1, 70.0, 64.9, 61.9,56, 52.2, 29.7, 20.4. MS (EI) $\mathrm{m} / z 389$ $(\mathrm{M}+1)^{+}$, HRMS calculated $(\mathrm{M}+\mathrm{H})^{+}$389.0865, found 389.0852, Anal. Calc. for $\mathrm{C}_{19} \mathrm{H}_{21} \mathrm{BrN}_{2} \mathrm{O}_{2}$ : C, 58.62; H, 5.44; N, 7.20; found: C, 58.60; H, 5.41; N, 7.28.

Methyl 4-bromo-3-((4-chlorophenyl)amino)-5-phenylpyrrolidine-2-carboxylate (7g). Yield: 80\%; Brown solid; $\delta_{\mathrm{H}}{ }^{1} \mathrm{H}$ NMR $\left(500 \mathrm{MHz}, \mathrm{CDCl}_{3}\right)$ 7.42-7.45 (m, 2H, ArH), 7.287.38 (m, 3H, ArH), 7.11 (t, J 6.5 Hz, 2H, ArH), 6.81-6.87 (m, 2H, ArH), 4.62 (d, J 5.5 Hz, $\left.1 \mathrm{H}, \mathrm{H}_{2}\right), 4.46\left(\mathrm{~d}, J 5.5 \mathrm{~Hz}, 1 \mathrm{H}, \mathrm{H}_{5}\right), 4.37\left(\mathrm{bs}, 1 \mathrm{H}, \mathrm{H}_{3}\right), 4.08\left(\mathrm{dd}, J 6.0\right.$ \& $\left.3.0 \mathrm{~Hz}, 1 \mathrm{H}, \mathrm{H}_{4}\right)$, $3.65\left(\mathrm{~s}, 3 \mathrm{H}, \mathrm{COOCH}_{3}\right) . \delta_{\mathrm{C}} \mathrm{NMR}\left(75 \mathrm{MHz}_{2} \mathrm{CDCl}_{3}\right) \delta 169.7,145.2,139.0,129.6,129.1$, $128.7,125.1,116.7,114.3,70.9,64.7,60.9,56.1,53.0$. MS (EI) $m / z ~ 409(\mathrm{M}+1)^{+}$, HRMS calculated $(\mathrm{M}+\mathrm{H})^{+}$409.0318, found 409.0313, Anal. Calc. for $\mathrm{C}_{18} \mathrm{H}_{18} \mathrm{BrClN}_{2} \mathrm{O}_{2}$ : C, 52.77; H, 4.43; N, 6.84; found: C, 52.73; H, 4.39; N, 6.87.

Methyl 4-bromo-3-((4-methoxyphenyl)amino)-5-phenylpyrrolidine-2-carboxylate (7h). Yield: 82\%; Brown solid; $\delta_{\mathrm{H}}{ }^{1} \mathrm{H}$ NMR (500 MHz, $\left.\mathrm{CDCl}_{3}\right)$ 7.47-7.49 (m, 2H, ArH), 7.28-7.42 (m, 3H, ArH), 7.22-7.23 (m, 2H, ArH), 7.04-7.06 (m, 2H, ArH), 4.61 (d, J 6.0 $\left.\mathrm{Hz}, 1 \mathrm{H}, \mathrm{H}_{2}\right), 4.46\left(\mathrm{~d}, J 5.5 \mathrm{~Hz}, 1 \mathrm{H}, \mathrm{H}_{5}\right), 4.34$ (bs, 1H, H3), 4.09 (dd, J 6.5 \& $3.5 \mathrm{~Hz}, 1 \mathrm{H}$, $\left.\mathrm{H}_{4}\right), 3.69\left(\mathrm{~s}, 3 \mathrm{H}, \mathrm{OCH}_{3}\right), 3.52\left(\mathrm{~s}, 3 \mathrm{H}, \mathrm{COOCH}_{3}\right) . \delta \mathrm{C} \mathrm{NMR}\left(75 \mathrm{MHz}, \mathrm{CDCl}_{3}\right) \delta 171.8,146.0$, 139.0, 129.1, 128.7, 128.1, 126.6, 118.4, 114.3, 70.9, 64.7, 61.3, 57.5, 56.1, 52.7. MS (EI) $\mathrm{m} / \mathrm{z} 405(\mathrm{M}+1)^{+}$, HRMS calculated $(\mathrm{M}+\mathrm{H})^{+}$405.0814, found 405.0806, Anal. Calc. for $\mathrm{C}_{19} \mathrm{H}_{21} \mathrm{BrN}_{2} \mathrm{O}_{3}$ : C, 56.31; H, 5.22; N, 6.91; found: C, 56.29; H, 5.17; N, 6.96.

Ethyl 4-iodo-5-phenyl-3-(phenylamino)pyrrolidine-2-carboxylate (7i). Yield: 86\%; White solid; $\delta_{\mathrm{H}}{ }^{1} \mathrm{H}$ NMR $\left(500 \mathrm{MHz}, \mathrm{CDCl}_{3}\right)$ 7.28-40 (m, 5H, ArH), 7.06-7.12 (m, 2H, ArH), 6.76 (t, J 7.5 Hz, 1H, ArH), 6.62 (d, J 7.5 Hz, 2H, ArH), $4.70\left(\mathrm{~d}, J 6.0 \mathrm{~Hz}, 1 \mathrm{H}, \mathrm{H}_{2}\right)$, $4.46\left(\mathrm{~m}, 2 \mathrm{H}, \mathrm{H}_{3} \& \mathrm{H}_{4}\right), 4.17\left(\mathrm{~m}, 2 \mathrm{H}, \mathrm{CH}_{2}\right), 4.08\left(\mathrm{~d}, J 7.0 \mathrm{~Hz}, 1 \mathrm{H}, \mathrm{H}_{5}\right), 1.27$ (t, J 7.5 Hz, 3H, $\left.\mathrm{CH}_{2} \mathrm{CH}_{3}\right) . \delta_{\mathrm{C}} \mathrm{NMR}\left(75 \mathrm{MHz}, \mathrm{CDCl}_{3}\right) \delta 170.7,145.6,139.4,129.4,128.7,128.3,127.0$, 
118.7, 113.6, 71.2, 66.1, 61.3, 60.3, 29.3, 14.5. MS (EI) $m / z 437(\mathrm{M}+1)^{+}$, HRMS calculated $(\mathrm{M}+\mathrm{H})$ 437.0726, found 437.0720, Anal. Calc. for $\mathrm{C}_{19} \mathrm{H}_{21} \mathrm{IN}_{2} \mathrm{O}_{2}$ : C, 52.31; H, 4.85; N, 6.42; found: C, 52.27; H, 4.79; N, 6.47.

Ethyl 4-iodo-5-phenyl-3-(p-tolylamino)pyrrolidine-2-carboxylate (7j). Yield: 82\%; White solid; $\delta_{\mathrm{H}}{ }^{1} \mathrm{H}$ NMR $\left(500 \mathrm{MHz}, \mathrm{CDCl}_{3}\right)$ 7.35-7.49 (m, 4H, ArH), 7.22-7.28 (m, 3H, ArH), 7.05 (d, J 8.5 Hz, 2H, ArH), $4.68\left(\mathrm{~d}, J 7.0 \mathrm{~Hz}, 1 \mathrm{H}, \mathrm{H}_{2}\right), 4.39-4.50\left(\mathrm{~m}, 2 \mathrm{H}, \mathrm{H}_{3}\right.$ \& $\left.\mathrm{H}_{4}\right)$, 4.10-4.18 (m, 3H, $\left.\mathrm{CH}_{2} \& \mathrm{H}_{5}\right), 2.27\left(\mathrm{~s}, 3 \mathrm{H}, \mathrm{CH}_{3}\right), 1.28\left(\mathrm{t}, J 7.5 \mathrm{~Hz}, 3 \mathrm{H}, \mathrm{CH}_{2} \mathrm{CH}_{3}\right) . \delta_{\mathrm{C}} \mathrm{NMR}$ $\left(75 \mathrm{MHz} \mathrm{CDCl}_{3}\right) \delta 171.4,143.5,139.4,129.4,128.7,128.1,126.3,120.8,114.3,71.2$, $66.5,62.0,60.3,33.8,20.7,13.8$. MS (EI) $\mathrm{m} / \mathrm{z} 451(\mathrm{M}+1)^{+}$, HRMS calculated $(\mathrm{M}+\mathrm{H})^{+}$ 451.0882, found 451.0879, Anal. Calc. for $\mathrm{C}_{20} \mathrm{H}_{23} \mathrm{IN}_{2} \mathrm{O}_{2}$ : C, 53.34; H, 5.15; N, 6.22; found: C, 53.31; H, 5.10; N, 6.27 .

Ethyl 4-bromo-5-phenyl-3-(phenylamino)pyrrolidine-2-carboxylate (7k). Yield: 73\%; Brown solid; $\delta_{\mathrm{H}}{ }^{1} \mathrm{H}$ NMR $\left(500 \mathrm{MHz}, \mathrm{CDCl}_{3}\right) 7.50(\mathrm{~m}, 2 \mathrm{H}, \mathrm{ArH}), 7.32-7.48$ (m, 5H, ArH), 7.23-7.28 (m, 2H, ArH), 7.02 (t, J 7.5 Hz, 1H, ArH), 4.61 (d, J $\left.5.5 \mathrm{~Hz}, 1 \mathrm{H}, \mathrm{H}_{2}\right), 4.46$ (d, J $\left.5.5 \mathrm{~Hz}, 1 \mathrm{H}, \mathrm{H}_{5}\right), 4.39$ (bs, $\left.1 \mathrm{H}, \mathrm{H}_{3}\right), 4.06-4.13\left(\mathrm{~m}, 3 \mathrm{H}, \mathrm{CH}_{2} \& \mathrm{H}_{4}\right), 1.19(\mathrm{t}, J 7.5 \mathrm{~Hz}, 3 \mathrm{H}$, $\left.\mathrm{CH}_{2} \mathrm{CH}_{3}\right) . \delta \mathrm{C}$ NMR $\left(75 \mathrm{MHz}, \mathrm{CDCl}_{3}\right) \delta 171.1,145.2,139.4,129.4,129.1,127.8,126.3$, 118.7, 114.0, 69.9, 64.4, 61.6, 60.6, 56.9, 14.5. MS (EI) $m / z 390(\mathrm{M}+1)^{+}$, HRMS calculated $(\mathrm{M}+\mathrm{H})^{+}$389.0865, found 389.0855, Anal. Calc. for $\mathrm{C}_{19} \mathrm{H}_{21} \mathrm{BrN}_{2} \mathrm{O}_{2}$ : C, 58.62; H, 5.44; N, 7.20; found: C, 58.59; H, 5.36; N, 7.24.

Ethyl 4-bromo-5-phenyl-3-(p-tolylamino)pyrrolidine-2-carboxylate (71). Yield: $81 \%$; Brown solid; $\delta_{\mathrm{H}}{ }^{1} \mathrm{H}$ NMR $\left(500 \mathrm{MHz}, \mathrm{CDCl}_{3}\right)$ 7.28-7.49 (m, 5H, ArH), 7.22 (m, 2H, ArH), 7.05 (m, 2H, ArH), 4.59 (d, J $\left.6.0 \mathrm{~Hz}, 1 \mathrm{H}, \mathrm{H}_{2}\right), 4.47$ (d, J 6.0 Hz, 1H, H5), $4.36\left(\mathrm{bs}, 1 \mathrm{H}, \mathrm{H}_{3}\right)$, 4.08-4.17 (m, 3H, $\left.\mathrm{CH}_{2} \& \mathrm{H}_{4}\right), 2.21\left(\mathrm{~s}, 3 \mathrm{H}, \mathrm{CH}_{3}\right), 1.28\left(\mathrm{t}, J 7.5 \mathrm{~Hz}, 3 \mathrm{H}, \mathrm{CH}_{2} \mathrm{CH}_{3}\right) . \delta_{\mathrm{C}} \mathrm{NMR}$ $\left(75 \mathrm{MHz}, \mathrm{CDCl}_{3}\right) \delta 170.1,143.4,139.8,129.7,129.4,128.7,128.1,126.6,114.3,70.3$, 64.7, 62.0, 60.6, 56.1 , 29.7, 21.5, 13.7. MS (EI) $\mathrm{m} / \mathrm{z} 404(\mathrm{M}+1)^{+}$, HRMS calculated $(\mathrm{M}+\mathrm{H})^{+}$ 403.1021, found 403.1014, Anal. Calc. for $\mathrm{C}_{20} \mathrm{H}_{23} \mathrm{BrN}_{2} \mathrm{O}_{2}: \mathrm{C}, 59.56 ; \mathrm{H}, 5.75 ; \mathrm{N}, 6.95$; found: C, 59.51; H, 5.73; N, 6.98.

Typical procedure for the preparation of 4,6-diaryl-3,6-diazabicyclo[3.1.0] hexane-2carboxylic acids (8). To a solution of compound 2 (30mg, 1 eq) in methanol/ethanol (5 $\mathrm{mL}), \mathrm{NaOMe} / \mathrm{NaOEt}(6.5 \mathrm{eq})$ was added and the reaction mixture was stirred at room temperature for $1 \mathrm{hr}$. Then the reaction mixture was heated up to $50{ }^{\circ} \mathrm{C}$ for 30 minutes. The progress of the reaction was monitored with the help of TLC. After completion of the reaction, the mixture was quenched with ice and $\mathrm{pH}$ adjust to 6-7. Now, the reaction mixture was concentrated under reduced pressure and purified via flash column chromatography using silica gel (100:200 mesh) in MeOH/DCM (1:9) as an elutent system to get compound 6 as a pure product.

4,6-Diphenyl-3,6-diazabicyclo[3.1.0]hexane-2-carboxylic acid (8a). Yield: 90\%; Brown solid; $\delta_{\mathrm{H}}{ }^{1} \mathrm{H}$ NMR (300 MHz, MeOD) 7.68 (dd, J $\left.8.4 \& 1.5 \mathrm{~Hz}, 2 \mathrm{H}, \mathrm{ArH}\right), 7.31-7.42$ (m, $3 \mathrm{H}, \mathrm{ArH}), 7.12(\mathrm{t}, J 7.8 \mathrm{~Hz}, 2 \mathrm{H}, \mathrm{ArH}), 6.58$ (d, J $7.8 \mathrm{~Hz}, 3 \mathrm{H}, \mathrm{ArH}), 4.10$ (d, J 1.8 Hz, 1H, 
$\left.\mathrm{H}_{2}\right), 3.68$ (d, J $\left.1.8 \mathrm{~Hz}, 1 \mathrm{H}, \mathrm{H}_{5}\right), 3.23$ (dd, J 4.5, 2.1 Hz, 1H, $\left.\mathrm{H}_{3}\right), 3.08$ (dd, J 4.5, $2.1 \mathrm{~Hz}, 1 \mathrm{H}$, $\left.\mathrm{H}_{4}\right) . \delta_{\mathrm{C}} \mathrm{NMR}\left(75 \mathrm{MHz}, \mathrm{DMSOD}_{6}\right) \delta 173.8,154.1,141.8,129.07,128.6,127.8,127.5$, 121.7, 120.9, 64.3, 63.6, 49.7, 49.0. MS (EI) $m / z 281(\mathrm{M}+1)^{+}$, HRMS calculated $(\mathrm{M}+\mathrm{H})^{+}$ 281.1290, found 281.1289, Anal. Calc. for $\mathrm{C}_{17} \mathrm{H}_{16} \mathrm{~N}_{2} \mathrm{O}_{2}$ : C, 72.84; H, 5.75; N, 9.99; found: C, 72.78; H, 5.71; N, 10.02 .

4-Phenyl-6-(p-tolyl)-3,6-diazabicyclo[3.1.0]hexane-2-carboxylic acid (8b). Yield: 88\%; Brown solid; $\delta_{\mathrm{H}}{ }^{1} \mathrm{H}$ NMR (500 MHz, MeOD) 7.51 (m, 2H, ArH), 7.25-7.48 (m, 3H, ArH), $7.03(\mathrm{~m}, 2 \mathrm{H}, \mathrm{ArH}), 6.70(\mathrm{~d}, J 7.5 \mathrm{~Hz}, 2 \mathrm{H}, \mathrm{ArH}), 4.09\left(\mathrm{~d}, J 2.0 \mathrm{~Hz}, 1 \mathrm{H}, \mathrm{H}_{2}\right), 3.65$ (d, J 2.0 $\left.\mathrm{Hz}, 1 \mathrm{H}, \mathrm{H}_{5}\right), 3.19$ (dd, J 4.0, $\left.2.0 \mathrm{~Hz}, 1 \mathrm{H}, \mathrm{H}_{3}\right), 3.08$ (dd, J 4.5, $\left.2.0 \mathrm{~Hz}, 1 \mathrm{H}, \mathrm{H}_{4}\right), 2.27(\mathrm{~s}, 3 \mathrm{H}$, $\left.\mathrm{CH}_{3}\right) . \delta_{\mathrm{C}} \mathrm{NMR}\left(75 \mathrm{MHz}, \mathrm{DMSO}-\mathrm{D}_{6}\right) \delta 172.8,154.5,141.7,129.1,128.5,127.5,127.4$, 121.8, 121.3, 64.3, 63.9, 49.8, 49.2, 21.4. MS (EI) $\mathrm{m} / z 295(\mathrm{M}+1)^{+}$, HRMS calculated $(\mathrm{M}+\mathrm{H})^{+}$295.1447, found 295.1440, Anal. Calc. for $\mathrm{C}_{18} \mathrm{H}_{18} \mathrm{~N}_{2} \mathrm{O}_{2}$ : C, 73.45; H, 6.16; N, 9.52; found: C, 73.38; H, 6.10; N, 9.54.

6-(4-Chlorophenyl)-4-phenyl-3,6-diazabicyclo[3.1.0]hexane-2-carboxylic acid (8c). Yield: 82\%; Brown solid; $\delta_{\mathrm{H}}{ }^{1} \mathrm{H}$ NMR (500 MHz, MeOD) 7.25-7.51 (m, 5H, ArH), 6.997.02 (m, 2H, ArH), 6.71-6.76 (m, 2H, ArH), 4.10 (d, J 2.0 Hz, 1H, H2), 3.64 (d, J 2.0 Hz, $\left.1 \mathrm{H}, \mathrm{H}_{5}\right), 3.20\left(\mathrm{dd}, J 4.5,2.0 \mathrm{~Hz}, 1 \mathrm{H}, \mathrm{H}_{3}\right), 3.08\left(\mathrm{~m}, 1 \mathrm{H}, \mathrm{H}_{4}\right) . \delta_{\mathrm{C}} \mathrm{NMR}\left(75 \mathrm{MHz}, \mathrm{DMSO}-\mathrm{D}_{6}\right)$ $\delta 172.1,154.5,141.8,129.4,128.3,127.4,127.0,121.5,120.8,64.4,63.3,48.9,47.9 . \mathrm{MS}$ (EI) $m / z 315(\mathrm{M}+1)^{+}$, HRMS calculated $(\mathrm{M}+\mathrm{H})^{+}$315.0900, found 315.0891, Anal. Calc. for $\mathrm{C}_{17} \mathrm{H}_{15} \mathrm{ClN}_{2} \mathrm{O}_{2}$ : C, 64.87; H, 4.80; N, 8.90; found: C, 64.85; H, 4.85; N, 8.96.

6-(4-Methoxyphenyl)-4-phenyl-3,6-diazabicyclo[3.1.0]hexane-2-carboxylic acid (8d). Yield: 85\%; Brown solid; $\delta_{\mathrm{H}}{ }^{1} \mathrm{H}$ NMR (500 MHz, MeOD) 7.23-7.53 (m, 5H, ArH), 6.74$7.02(\mathrm{~m}, 4 \mathrm{H}, \mathrm{ArH}), 4.10\left(\mathrm{~d}, J 1.5 \mathrm{~Hz}, 1 \mathrm{H}, \mathrm{H}_{2}\right), 3.66\left(\mathrm{~d}, J 1.5 \mathrm{~Hz}, 1 \mathrm{H}, \mathrm{H}_{5}\right), 3.23$ (dd, $J$ 4.5, $\left.2.0 \mathrm{~Hz}, 1 \mathrm{H}, \mathrm{H}_{3}\right), 3.19\left(\mathrm{~s}, 3 \mathrm{H}, \mathrm{OCH}_{3}\right) 3.07\left(\mathrm{dd}, J 4.5 \& 2.0 \mathrm{~Hz}, 1 \mathrm{H}, \mathrm{H}_{4}\right) . \delta_{\mathrm{C}} \mathrm{NMR}(75 \mathrm{MHz}$, DMSO-D $) \delta 173.8,154.2,141.5,129.8,128.7,127.7,127.4,121.9,120.4,64.4,63.0,55.8$, 49.9, 48.9. MS (EI) $m / z 311(\mathrm{M}+1)^{+}$, HRMS calculated $(\mathrm{M}+\mathrm{H})^{+} 311.1396$, found 310.1388 , Anal. Calc. for $\mathrm{C}_{18} \mathrm{H}_{18} \mathrm{~N}_{2} \mathrm{O}_{3}$ : C, 69.66; H, 5.85; N, 9.03; found: C, 69.63; H, 5.78; N, 9.08.

\section{Acknowledgements}

The financial support from Department of Science and Technology (DST), New Delhi, under Scheme No:- SB/FT/CS-079/2012 and Board of Research in Nuclear Sciences (BRNS), India (Scheme No. 2013/37C/11/BRNS/198) is highly acknowledged. Mr. Yogesh Kumar acknowledge the UGC Scheme RGNF (Award No:- F1-17.1/2011-12/RGNF-SC-PUN-2309/(SA-III/Website)). I. K. Gujral Punjab Technical University (PTU), Kapurthala is acknowledged for providing research facilities. 


\section{References}

1. Alcaide, B.; P. Almendros, Curr. Med. Chem. 2004, 11, 1921. http://dx.doi.org/10.2174/0929867043364856

2. Ojima, I. Adv. Asym. Synth. 1995, 1, 95.

3. A. Kamath, I. Ojima, Tetrahedron 2012, 60, 10640. http://dx.doi.org/10.1016/j.tet.2012.07.090

4. Ojima, I. Acc. Chem. Res. 1995, 28, 383. http://dx.doi.org/10.1021/ar00057a004

5. Ojima, I.; Das, M. J. Nat. Prod. 2009, 72, 554. http://dx.doi.org/10.1021/np8006556

6. Geng, X.; Miller, M.; Lin, S.; Ojima, I. Org. Lett. 2003, 5, 3733. http://dx.doi.org/10.1021/ol0354627

7. Alcaide, B.; Almendros, P.; Alonso, J. M.; Aly, M. F. Chem. Eur. J. 2003, 9, 3415. http://dx.doi.org/10.1002/chem.200304712

8. Alcaide, B.; Almendros, P.; Aragoncillo, C. Chem. Eur. J. 2002, 8, 3646. http://dx.doi.org/10.1002/1521-3765(20020816)8:16<3646::AID-CHEM3646>3.0.CO;2-M

9. Singh, P.; Bhargava, G.; Kumar, V.; Mahajan, M. P. Tetrahedron Lett. 2010, 51, 4272. http://dx.doi.org/10.1016/j.tetlet.2010.06.038

10. Anand, A.; Bhargava, G.; Kumar, V.; Mahajan, M. P. Tetrahedron Lett. 2010, 51, 2312. http://dx.doi.org/10.1016/j.tetlet.2010.02.127

11. Raj, R.; Mehra, V.; Singh, P.; Kumar, V.; Bhargava, G.; Mahajan, M. P.; Handa, S.; Slaughter, L. M. Eur. J. Org. Chem. 2011, 14, 2697. http://dx.doi.org/10.1002/ejoc.201100130

12. Singh, P.; Raj, R.; Bhargava, G.; Hendricks, D. T.; Handa, S.; Slaughter, L. M.; Kumar, V. Eur. J. Med. Chem. 2012, 58, 513. http://dx.doi.org/10.1016/j.ejmech.2012.10.049

13. Davis, F. A.; Xu, H.; Wu, Y.; Zhang, J. Org. Lett. 2006, 8, 2273. http://dx.doi.org/10.1021/o1060521c

14. Rogge ,B.; Itagaki ,Y.; Fishkin , N.; Levi ， E.; Rühl „„ R.; Yi ,S.-S.; Nakanishi ， K.; Hammerling, U. J. Nat. Prod. 2005, 68, 1536. http://dx.doi.org/10.1021/np0496791

15. Fache, F.; Schultz, E.; Tomasino, M. L.; Lemaire, M. Chem. Rev. 2000, 100, 2159. http://dx.doi.org/10.1021/cr9902897

16. Hata, T.; Sano, Y.; Sugawara, R.; Matsumae, A.; Kanamori, K.; Shima, T.; Hoshi, T. J. Antibiot. 1956, 9, 141.

17. Sim, T. B.; Kang, S. H.; Lee, K. S.; Lee, W. K.; Yun, H.; Dong, Y.; Ha, H.-J. J. Org. Chem. 2003, 68, 104.

http://dx.doi.org/10.1021/jo0261911 
18. Park, C. S.; Kim, M. S.; Sim, T. B.; Pyun, D. K.; Lee, C. H.; Choi, D.; Lee, W. K.; Chang, J.W.; Ha, H.-J. J. Org. Chem. 2003, 68, 43. http://dx.doi.org/10.1021/jo0255451

19. Zwanenburg, B.; ten Holte, P. Stereoselective Heterocyclic Synthesis III; Metz, P., Ed.; Topics in Current Chemistry 216; Springer-Verlag: Berlin, Heidelberg, 2001; p 93.

20. McCoull, W.; Davis, F. A. Synthesis 2000, 10, 1347. http://dx.doi.org/10.1055/s-2000-7097

21. Sweeney, J. B. Chem. Soc. Rev. 2002, 31, 247. http://dx.doi.org/10.1039/B006015L

22. Brown, F. Vaccine 2002, 20, 322. http://dx.doi.org/10.1016/S0264-410X(01)00342-5

23. Louw, A.; Swart, P.; Allie, F. Biochem. Pharmacol. 2000, 59, 167. http://dx.doi.org/10.1016/S0006-2952(99)00302-0

24. Dvorakova, K.; Payne, C. M.; Tome, M. E.; Briehl, M. M.; McClure, T.; Dorr, R. T. Biochem. Pharmacol. 2000, 60, 749. http://dx.doi.org/10.1016/S0006-2952(00)00380-4

25. Furmeier, S.; Metzger, J. O. Eur. J. Org. Chem. 2003, 649. http://dx.doi.org/10.1002/ejoc.200390105

26. Kumar, Y.; Singh, P.; Bhargava, G. Synlett 2015, 26, 363. http://dx.doi.org/10.1055/s-0034-1379505

27. Bains, D.; Kumar, Y.; Singh, P.; Bhargava, G. J. Heterocyclic Chem. 2016, 53, 1665, DOI 10.1002/jhet.2465. http://dx.doi.org/10.1002/jhet.2465

28. B. Kuila, Kumar, Y.; Mahajan, D.; Kumar, K.; Singh, P.; Bhargava, G. RSC Adv. 2016, 6, 57485-57489. http://dx.doi.org/10.1039/C6RA10021J

29. Kumar, Y.; Kuila, B.; Mahajan, D.; Singh, P.; Mohapatra, B.; Bhargava, G. Tetrahedron Lett. 2014, 55, 2793. http://dx.doi.org/10.1016/j.tetlet.2014.02.105

30. Paukner, S.; Hesse, L.; Prezelj, A.; Solmajer, T.; Urleb, U. Antimicrob. Agents Chemother. 2009, 53, 505. http://dx.doi.org/10.1128/AAC.00085-08

31. Silver, L. L. Expert Opin. Ther. Patents, 2007, 17, 1175. http://dx.doi.org/10.1517/13543776.17.9.1175

32. Herdeis, C.; Aschenbrenner, A.; Kirfel, A.; Schwabenlainder, F. Tetrahedron: Asymm. 1997, 8, 2421-2432. http://dx.doi.org/10.1016/S0957-4166(97)00261-9

33. Steen, F. H. V.; Koten, G. V. Tetrahedron 1991, 47, 7503 and reference cited therein. http://dx.doi.org/10.1016/S0040-4020(01)88276-4 